Provided for non-commercial research and educational use only. Not for reproduction or distribution or commercial use.

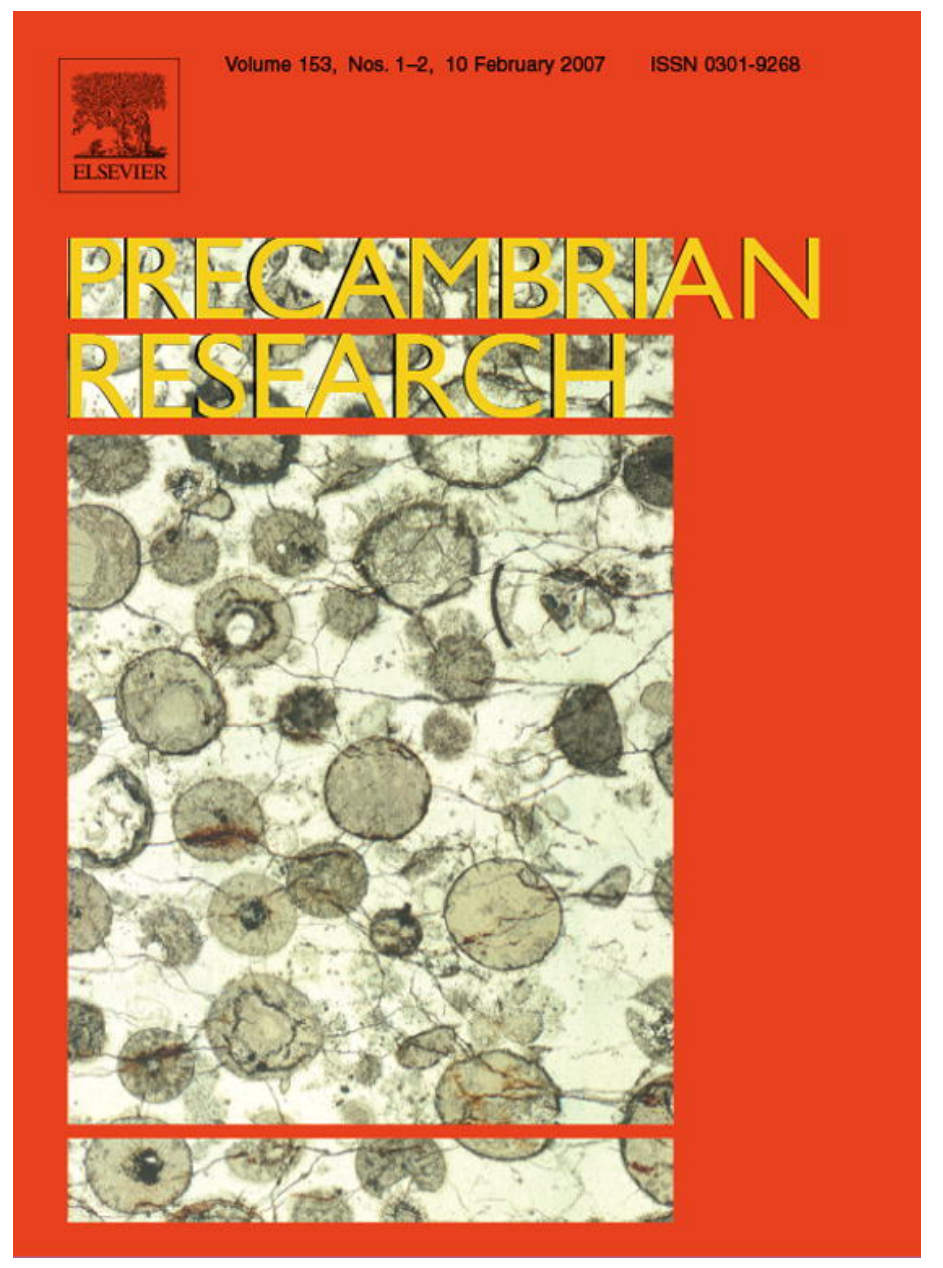

This article was originally published in a journal published by Elsevier, and the attached copy is provided by Elsevier for the author's benefit and for the benefit of the author's institution, for non-commercial research and educational use including without limitation use in instruction at your institution, sending it to specific colleagues that you know, and providing a copy to your institution's administrator.

All other uses, reproduction and distribution, including without limitation commercial reprints, selling or licensing copies or access,

or posting on open internet sites, your personal or institution's website or repository, are prohibited. For exceptions, permission may be sought for such use through Elsevier's permissions site at: 


\title{
Geochemical constraints of the petrogenesis of the O'okiep Koperberg Suite and granitic plutons in Namaqualand, South Africa: A crustal source in Namaquan (Grenville) times
}

\author{
Jean-Clair Duchesne ${ }^{\mathrm{a}, *}$, Jacqueline Vander Auwera ${ }^{\mathrm{a}}$, \\ Jean-Paul Liégeois ${ }^{\mathrm{a}, \mathrm{b}}$, Erika S. Barton ${ }^{\mathrm{c}}$, Tom N. Clifford ${ }^{\mathrm{d}}$ \\ a Département de Géologie, Unité de Recherche en Pétrologie et Géochimie Endogènes, Université de Liège, Sart Tilman, Belgium \\ ${ }^{\mathrm{b}}$ Africa Museum, Section de Géologie Isotopique, Tervuren, Belgium \\ ${ }^{\mathrm{c}}$ Hugh Allsopp Laboratory, Economic Geology Research Institute, School of Geosciences, \\ University of the Witwatersrand, Johannesburg, South Africa \\ ${ }^{\mathrm{d}}$ School of Geosciences, University of the Witwatersrand, Johannesburg, South Africa
}

Received 25 February 2006; received in revised form 12 November 2006; accepted 20 November 2006

\begin{abstract}
The Namaquan (Grenville) Orogeny (late Mesoproterozoic) in the O'okiep District is characterized by two tectono-magmatic episodes: the O'okiepian Episode (1210-1180 Ma) with the intrusion of batholitic granites, and the Klondikean Episode (1040-1020 Ma), which includes the intrusion of the copper-bearing Koperberg Suite and the Rietberg Granite. This study focuses on the geochemistry (major and trace elements, $\mathrm{Sr}$ and $\mathrm{Nd}$ isotopes) of intrusive rocks of the O'okiep terrane to provide better constraints on their source rock characteristics and on their petrogenesis. The O'okiepian Granites belong to the K-rich granite kindred, with shoshonitic affinities. The Concordia Granite results from dehydration melting of a pelitic source. The Rietberg Granite shows geochemical similarities with post-collisional magmatic series. The anorthosites and related rocks of the Koperberg Suite are cumulates; their REE distribution is controlled by their apatite content. A new rock type, jotunite, has been identified in the Koperberg Suite; it is analogous to the Rogaland chilled jotunite, a characteristic which gives strong evidence that the Koperberg rocks belong to the massif-type anorthosite suite. Inversion modelling of plagioclase REE compositions from anorthosite permits the reconstruction of melt compositions and places constraints on the melting process and on the characteristics of the source rocks. The occurrence of jotunite in the Koperberg Suite is strong additional evidence for a crustal source, because jotunite is produced by remelting of gabbronorite under dry conditions. The various intrusions in the Koperberg Suite show distinct isotopic signatures, which resulted from isotopic heterogeneities of the crustal source and from minor contamination with the country rocks. The characteristic negative $\varepsilon_{\mathrm{Nd}(1030 \mathrm{Ma})}$ values ( -5 to -11$)$ can be explained by remelting at $1030 \mathrm{Ma}$ of a 1900 -Ma-old oceanic crust protolith with an enriched MORB REE distribution. The large range in Sr initial ratios (0.709-0.748) may reflect hydrothermal alteration of the oceanic crust, a process which may also explain the $\mathrm{Cu}$ enrichment. The Koperberg intrusions were produced by forceful injection of cumulate crystal mush, which were differentiated in deeper magma chambers or in conduits.
\end{abstract}

(c) 2006 Elsevier B.V. All rights reserved.

Keywords: Granite plutonism; Anorthosite; Jotunite; $\mathrm{Cu}$ sulphides; Namaquan orogeny; $\mathrm{Sr}$ and $\mathrm{Nd}$ isotopes

\footnotetext{
* Corresponding author. Tel.: +32 4 3662255; fax: +32 43662921 .

E-mail address: jc.duchesne@ulg.ac.be (J.-C. Duchesne).
}

\section{Introduction}

The O'okiep intrusive rocks of Namaqualand, South Africa, include the Koperberg Suite and a number of 
important granite suites which intrude a basement of crystalline rocks, made up of granite gneiss with remnants of supracrustal rocks. These crystalline rocks were deformed and metamorphosed during the Namaquan (=Grenville) Orogeny (late Mesoproterozoic) (Clifford et al., 1981, 2004). The Koperberg Suite includes Cubearing sulphide deposits that have been mined for 150 years, and because of its economic importance, the O'okiep District has been the subject of detailed studies for the last 50 years (see e.g. Gibson et al., 1996). A number of recent publications have been devoted to the timing and interpretation of metamorphic and intrusive events (Clifford et al., 1995, 2004; Gibson et al., 1996; Robb et al., 1999). Fewer studies have, however, been concerned with the geochemistry of the intrusive rocks (Conradie and Schoch, 1988; Brandriss and Cawthorn, 1996; Van Zwieten et al., 1996; Geringer et al., 1998).

Several granites, including the Concordia and Kweekfontein Granites, were emplaced around $12000 \mathrm{Ma}$ (Clifford et al., 2004) and were, in turn, intruded at $c a$. $1030 \mathrm{Ma}$ (Clifford et al., 1995, 2004; Robb et al., 1999) by the Koperberg Suite of rocks, consisting of andesine anorthosite and various members of the charnockitic suite (sensu Streckeisen, 1974). In the present work, we show that most Koperberg Suite rocks are cumulates and we focus on the jotunite rocks that have the characteristics of melts which can be parental to andesine anorthosite and related rocks (Duchesne, 1990).
The isotopic characteristics of the crustal source of this parental magma (Clifford et al., 1995; Geringer et al., 1998) may be explained by the melting of a mafic (gabbronoritic) rock, a process supported by experimental work (Longhi et al., 1999; Longhi, 2005); this protolith was an hydrothermally altered oceanic crust with associated $\mathrm{Cu}$-sulphide deposits. Finally, the overall K-rich nature of the enclosing granitoids, whatever their age and evolution, is compatible with a post-collisional geodynamic setting (Liégeois et al., 1998).

\section{Regional setting}

The Namaquan orogenic belt in north-western South Africa and southern Namibia is made up of a series of terranes which have been grouped in subprovinces (Fig. 1; Hartnady et al., 1985): (1) the Gordonia subprovince, (2) the Richtersveld subprovince, and (3) the Bushmanland subprovince. The Gordonia subprovince is composed of several terranes between the Kaapvaal craton and the other two subprovinces. Among them the Areachap terrane, made up of greenstones generated $c a$. $1300 \mathrm{Ma}$ ago (Cornell et al., 1990), is the only juvenile terrane in the Namaquan orogen. The Richtersveld subprovince is interpreted as an Eburnian, 1700-2000Ma-old island arc complex remobilized along an active continental margin (Reid and Barton, 1983; Hartnady et al., 1985). Finally the Bushmanland subprovince com-

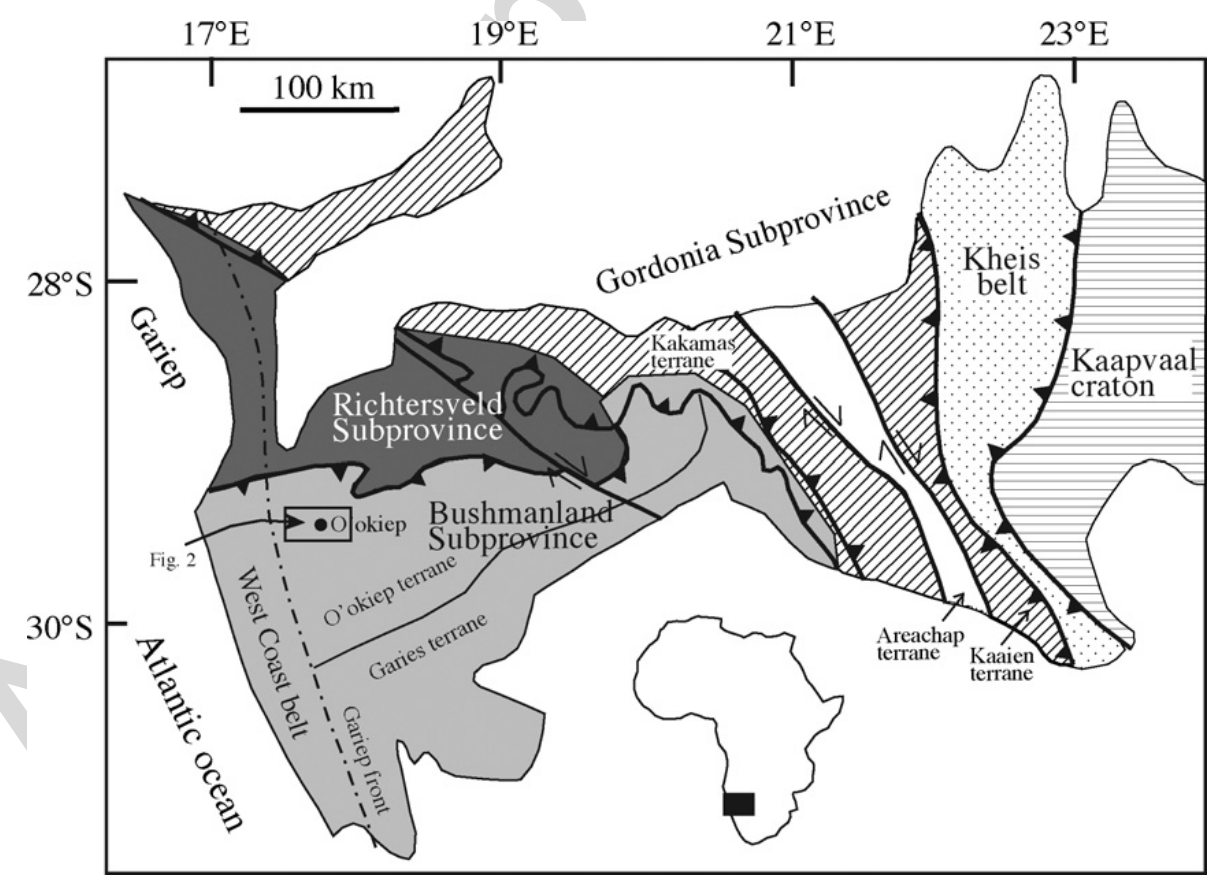

Fig. 1. Terrane map of the Namaquan orogen modified after Hartnady et al. (1985) and Thomas et al. (1994). The Gordonia subprovince (eastern Namaqua), which possesses rocks with active margin characteristics, the Richtersveld subprovince, made of preserved 2000-1700 Ma rocks, and the Bushmanland subprovince (south-western Namaqua) made also of an early Palaeoproterozoic basement but heavily reactivated during the Namaquan orogeny (1200-800 Ma). 
prises two terranes, the O'okiep and Garies terranes (Hartnady et al., 1985), which essentially differ by the absence of the Koperberg Suite in the latter. The northern boundary with the Bushmanland subprovince is marked by a major mylonitic ductile shear zone along which the Richtersveld subprovince has been thrust below the Bushmanland subprovince.

The geology of the O'okiep District has been summarized by Lombaard (1986). The oldest elements are Palaeoproterozoic supracrustal rocks that include the Khurisberg Subgroup of metasediments (Fig. 2), and the Lammerhoek Subgroup that is composed largely of quartz-feldspar-biotite granulite and gneiss with intercalations of various metasedimentary rocks (Clifford et al., 1981). The supracrustal rocks pre-date the widespread intrusion of granite at $c a$. 1200 Ma now represented by the foliated granite gneiss of the Gladkop and Little Namaqualand Suites, and the intrusion of the younger granites which include: (1) a sheet, up to $1500 \mathrm{~m}$ thick, of the garnetiferous Concordia Granite that is strongly lineated towards the base; (2) weakly foliated fine-grained sill- or dyke-like bodies of the Kweekfontein Granite; (3) the generally sheet-like porphyritic Rietberg Granite that is devoid of lineation and foliation (Lombaard, 1986). U-Pb SHRIMP ages on zircon for these intrusives have shown that the granites belong to two distinct intrusive episodes (Clifford et al., 2004): the Concordia $(1206 \pm 16 \mathrm{Ma})$ and Kweekfontein $(1186 \pm 15 \mathrm{Ma})$
Granites were intruded contemporaneously at about 1190 Ma during the O'okiepian Episode, whereas the Rietberg Granite (1035 $\pm 15 \mathrm{Ma})$ was contemporaneous with the emplacement of the Koperberg Suite ( $c a$. $1035 \mathrm{Ma}$ ) and belongs to the Klondikean Episode.

The fundamental $\left(\mathrm{D}_{2}\right)$ structure of the O'okiep District reflects reworking of intrafolial folds $\left(\mathrm{F}_{1}\right)$ and can be described in terms of major recumbent $\left(\mathrm{F}_{2}\right)$ folding (Clifford et al., 1975) that is recorded in the Concordia Granite. The principal regional granulite facies metamorphism $\left(\mathrm{M}_{2}\right)$ accompanied and outlasted this $\mathrm{D}_{2}$ tectonism. The open folding $\left(\mathrm{F}_{3}\right)$ post-dated all these events but pre-dated the intrusion of the Koperberg Suite and the development of tight folds $\left(\mathrm{F}_{4}\right)-$ locally referred to as "steep-structures" - and pipelike bodies of megabreccia (Lombaard and Schreuder, 1978; Lombaard, 1986). It is apparent that many of the bodies of the Koperberg Suite are detached remnants of intrusions that were boudinaged during late "steep structure" tectonism; and Kisters et al. (1994) have claimed that these intrusions were coeval with granulite facies metamorphism and ductile deformation $\left(D_{3}\right)$ of the country-rock gneiss.

\section{Previous work}

The regionally extensive (Fig. 2) Concordia Granite, and the Kweekfontein and Rietberg Granites have essen-

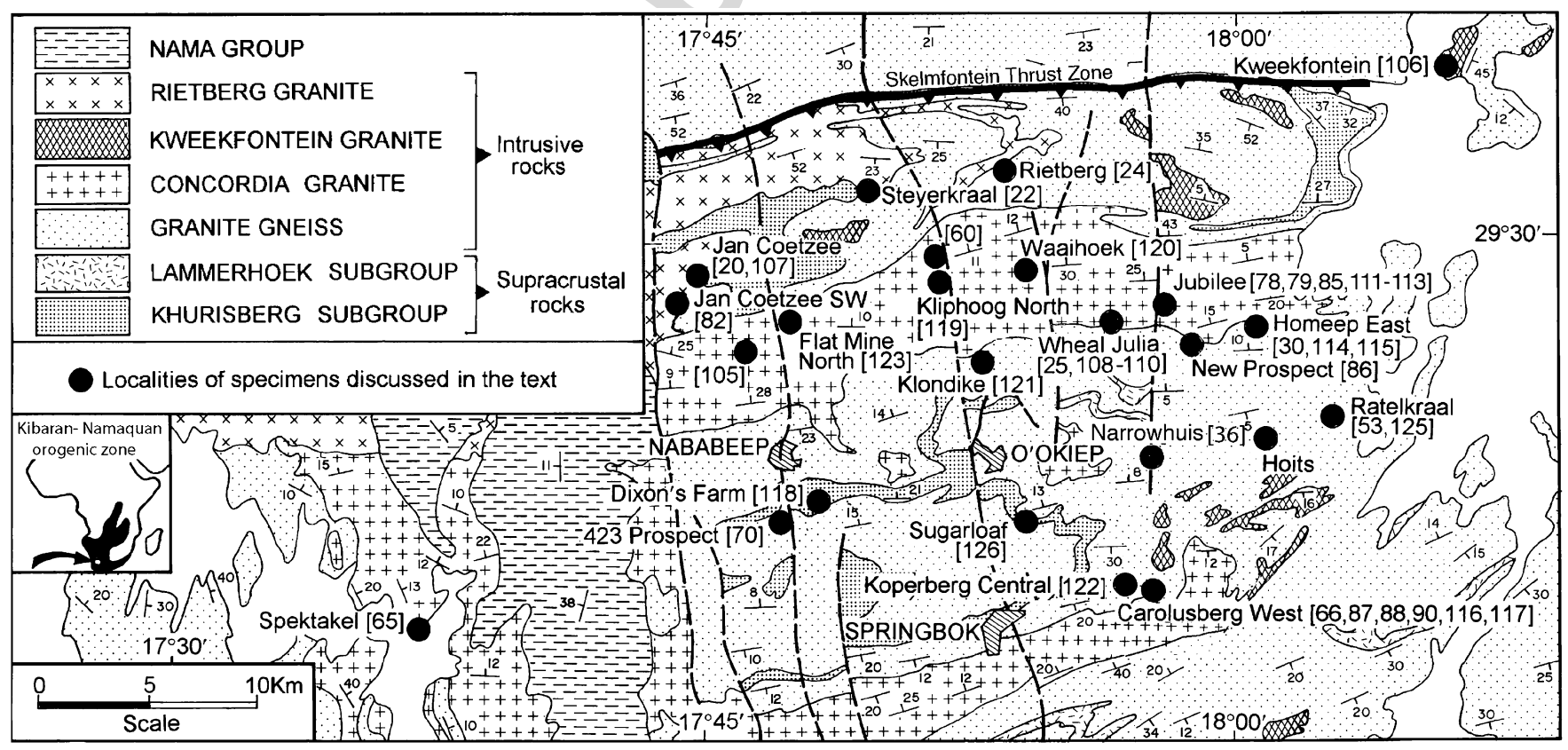

Fig. 2. Generalized geological map of the O'okiep District showing the location of the samples in this work. Granite gneiss includes the Gladkop and Little Namaqualand Suites (see Lombaard, 1986). The Koperberg Suite is not shown because of the small size of the intrusions. These crystalline rocks are unconformably overlain by the sediments of the Late Proterozoic-Early Palaeozoic Nama Group. Strike and dip of foliation are locally indicated. Adapted from Clifford et al. (2004). 
tially the same mineralogy with two feldspars, and biotite as the main mafic mineral. Of these, the Concordia Granite and related aplitic and pegmatitic varieties have been studied in detail by Raith (1995) who interpreted them as a fractionated S-type granite; notable features are high contents in $\mathrm{U}$ and $\mathrm{Th}$ and the presence of garnet in the accessory minerals.

The Koperberg Suite intrusions constitute only $0.7 \%$ of the outcrop area of the O'okiep District (Lombaard and Schreuder, 1978), but contain all of the primary copper for which the area is famous. Some 1700 bodies of the suite are known in the district and they occur either as elongate bodies or irregular pipes that rarely exceed $200 \mathrm{~m}$ in diameter, or as dykes up to $100 \mathrm{~m}$ in width and generally less than $1 \mathrm{~km}$ in length, often associated with "steep structures"(Lombaard, 1986). The principal rock types of the suite are andesine anorthosite, biotite diorite, and hypersthene-bearing varieties, notably leuconorite, norite, melanorite, and hypersthenite. The suite is subdivided into an earlier series of andesine anorthosite and biotite diorite intruded by hypersthene-bearing rocks (Strauss, 1941). Except for the term biotite diorite, this terminology is consistent with the classification of charnockitic rocks (Streckeisen, 1974). In addition, glimmerite and magnetitite (generally composed of equal proportions of magnetite and hypersthene) are present together with tonalite.

The mineralogy of the Koperberg Suite is simple, and the compositions of the minerals record restricted ranges (Conradie and Schoch, 1986; Cawthorn and Meyer, 1993; Brandriss and Cawthorn, 1996; Van Zwieten et al., 1996). The rocks consist of varying proportions of antiperthitic andesine $\left(\mathrm{An}_{30-50}\right)$, hypersthene $\left(\mathrm{En}_{58-68}\right)$, Ti-rich biotite $(\mathrm{MgO} / \mathrm{MgO}+\mathrm{FeO}=0.59-0.64$, up to $5.9 \% \mathrm{TiO}_{2}$ ), and quartz (Stumpfl et al., 1976). Magnetite, ilmenite, and $\mathrm{Cu}$-sulphides occur in the more mafic rocks, while apatite and zircon are ubiquitous accessories.

The texture of the Koperberg Suite rocks is quite variable. Igneous lamination reflecting the orientation of prismatic hypersthene crystals is common in melanorite, hypersthenite and glimmerite, while gently dipping layering has been observed locally (Brandriss and Cawthorn, 1996). Van Zyl (1978) and Brandriss and Cawthorn (1996) suggested a cumulate character for the series. In addition anorthosite, leuconorite and biotite diorite display a mosaic of anhedral, partly granulated plagioclase and subhedral unoriented mafic minerals, while deformation leading to polygonization/granulation and recrystallization is conspicuous (McIver et al., 1983; Van Zwieten et al., 1996).
McIver et al. (1983) have suggested that the parent magma of the Koperberg Suite was a mantlederived magma that has undergone contamination by granite partial melts in the lower crust. Contamination/hybridization models have also been presented by Schoch and Conradie (1990), Boer et al. (1994), Brandriss and Cawthorn (1996), Van Zwieten et al. (1996) and Geringer et al. (1998). In contrast, Andreoli and Hart (1987) have speculated that the high level of $\mathrm{K}, \mathrm{REE}$ and $\mathrm{P}$ in the Koperberg Suite may reflect melting of an enriched mantle source. The mantle-dominated model has, however, been challenged by Clifford et al. (1995) who have demonstrated that the Koperberg Suite is characterized by a variety of crustal isotopic signatures. For an emplacement age of 1030 Ma notably high initial $\mathrm{Sr}$ isotope ratios (0.7061-0.7272), low $\varepsilon_{\mathrm{Nd}}(-9)$, and high $\mu_{2}$ (10.1) suggest that the source was probably an $1700 \mathrm{Ma}\left(T_{\mathrm{CHUR}}\right)$ to $2000 \mathrm{Ma}\left(T_{\mathrm{DM}}\right)$ old granulite facies lower crust, that is reflected by the oldest zircon cores dated 1800-1850 Ma (Clifford et al., 2004).

\section{Results}

The analytical techniques are described in Appendix 2 together with the mineral separation method.

\subsection{Major and trace elements}

\subsubsection{Granites and syenite}

Three samples of the Concordia Granite (Fig. 2 and Appendix 1), one sample from the Kweekfontein Granite, four samples of the Rietberg Granite and one sample of a syenite coeval with the Rietberg Granite (Clifford et al., 2004) have been investigated (Table 1). The Concordia Granite is $\mathrm{SiO}_{2}$-rich, slightly peraluminous, typically rich in $\mathrm{U}$ and $\mathrm{Th}$, with a small REE variation, average $\mathrm{Eu}$ negative anomaly of 0.41 , and $[\mathrm{La} / \mathrm{Yb}]_{n}$ ratio of 8. The Kweekfontein Granite (sample 106) is geochemically similar in major and trace elements to the Concordia Granite (Fig. 3), except for its drastically different REE content. The $(\mathrm{La} / \mathrm{Yb})_{n}$ ratio is much higher [92] than in the Concordia Granite [8-9], and there is virtually no $\mathrm{Eu}$ anomaly. Major and trace elements, reported in Table 1, are plotted in Figs. 3-4 together with two characteristic rock series. First, the Rogaland jotunite series, representing a liquid line of descent (Vander Auwera et al., 1998b), a well-documented case of dry fractional crystallization without any contamination of a jotunite magma. This magmatism is cogenetic with the anorthosite-mangerite-charnockite-(rapakivi) granite (AMCG) suite, and has A-type affinities (Duchesne and Wilmart, 1997). Second, the Tismana (Romanian 
Table 1

Whole-rock compositions of granitic rocks and syenite from the O'okiep district

\begin{tabular}{|c|c|c|c|c|c|c|c|c|c|}
\hline & \multicolumn{3}{|c|}{ Concordia Granite } & \multirow{2}{*}{$\begin{array}{l}\text { Kweekfontein } \\
\text { Granite } \\
\text { Sample } 106\end{array}$} & \multicolumn{4}{|c|}{ Rietberg Granite } & \multirow{2}{*}{$\begin{array}{l}\text { Syenite } \\
\text { Sample } 65\end{array}$} \\
\hline & Sample 25 & Sample 60 & Sample 105 & & Sample 107 & Sample 20 & Sample 22 & Sample 24 & \\
\hline \multicolumn{10}{|l|}{ Major element (\%) } \\
\hline $\mathrm{SiO}_{2}$ & 71.09 & 73.34 & 72.91 & 71.46 & 62.8 & 63.50 & 67.21 & 72.40 & 49.58 \\
\hline $\mathrm{TiO}_{2}$ & 0.07 & 0.21 & 0.14 & 0.24 & 0.92 & 0.72 & 0.44 & 0.31 & 2.04 \\
\hline $\mathrm{Al}_{2} \mathrm{O}_{3}$ & 15.35 & 14.09 & 13.41 & 13.76 & 15.6 & 14.92 & 14.61 & 14.16 & 15.09 \\
\hline $\mathrm{Fe}_{2} \mathrm{O}_{3} \mathrm{t}$ & 1.53 & 1.95 & 1.46 & 1.92 & 4.09 & 5.45 & 3.03 & 2.60 & 7.74 \\
\hline $\mathrm{MnO}$ & 0.04 & 0.05 & 0.03 & 0.01 & 0.07 & 0.09 & 0.04 & 0.04 & 0.14 \\
\hline $\mathrm{MgO}$ & 0.35 & 0.26 & 0.36 & 0.27 & 1.97 & 2.18 & 1.37 & 0.57 & 4.24 \\
\hline $\mathrm{CaO}$ & 1.15 & 1.22 & 1.23 & 1.41 & 2.22 & 2.67 & 1.79 & 1.28 & 6.98 \\
\hline $\mathrm{Na}_{2} \mathrm{O}$ & 3.26 & 2.97 & 3.26 & 2.53 & 3.15 & 3.06 & 3.51 & 3.24 & 2.02 \\
\hline $\mathrm{K}_{2} \mathrm{O}$ & 6.03 & 5.95 & 6.16 & 6.51 & 7.11 & 4.88 & 6.28 & 6.18 & 6.08 \\
\hline $\mathrm{P}_{2} \mathrm{O}_{5}$ & 0.06 & 0.08 & 0.04 & 0.07 & 0.70 & 0.82 & 0.39 & 0.12 & 1.99 \\
\hline LOI & 0.95 & & 0.46 & 0.95 & 1.71 & 1.79 & 1.28 & 0.71 & 4.52 \\
\hline Total & 99.88 & 100.12 & 99.46 & 99.13 & 100.34 & 100.08 & 99.95 & 101.61 & 100.42 \\
\hline \multicolumn{10}{|l|}{ Trace elements (ppm) } \\
\hline $\mathrm{U}$ & 66 & 24 & 13 & 7.4 & 1.2 & 2.6 & 2.3 & 6.2 & 1.1 \\
\hline Th & 80 & 88 & 86 & 93 & 3.6 & 37 & 67 & 99 & 9.1 \\
\hline $\mathrm{Zr}$ & 141 & 187 & 157 & 220 & 257 & 503 & 299 & 259 & 585 \\
\hline $\mathrm{Hf}$ & 4.8 & 6.8 & 5.9 & 6.8 & 7.3 & 16 & 8.8 & 8.6 & 15 \\
\hline $\mathrm{Nb}$ & 8 & 15 & 9 & 5 & 26 & 15 & 11 & 12 & 30 \\
\hline $\mathrm{Ta}$ & 0.77 & 0.61 & 0.47 & 0.31 & 1.71 & 1.40 & 0.59 & 1.03 & 1.80 \\
\hline $\mathrm{Rb}$ & 419 & 326 & 303 & 227 & 252 & 222 & 287 & 318 & 183 \\
\hline $\mathrm{Cs}$ & 2.7 & 1.2 & 2.0 & 0.7 & 3.0 & 3.9 & 2.0 & 3.3 & 1.7 \\
\hline $\mathrm{Sr}$ & 58 & 105 & 101 & 164 & 950 & 1045 & 242 & 135 & 1918 \\
\hline $\mathrm{Ba}$ & 388 & 486 & 497 & 866 & 3851 & 3170 & 1630 & 775 & 6064 \\
\hline $\mathrm{V}$ & 13 & 14 & 8 & 20 & 60 & 93 & 33 & 21 & 231 \\
\hline $\mathrm{Co}$ & 3 & 2 & 1 & 2 & 13 & 13 & 7 & 3 & 29 \\
\hline $\mathrm{Zn}$ & 18 & 39 & 16 & 26 & 49 & 72 & 48 & 71 & 263 \\
\hline $\mathrm{Ga}$ & 17 & 18 & 16 & 17 & 18 & 18 & 20 & 19 & 90 \\
\hline $\mathrm{Pb}$ & 64 & 68 & 66 & 59 & 52 & 62 & 53 & 47 & 45 \\
\hline $\mathrm{Y}$ & 42 & 49 & 56 & 6.6 & 41 & 61 & 42 & 34 & 59 \\
\hline $\mathrm{La}$ & 52 & 75 & 78 & 80 & 61 & 106 & 282 & 125 & 208 \\
\hline $\mathrm{Ce}$ & 105 & 156 & 151 & 146 & 142 & 219 & 540 & 266 & 472 \\
\hline $\operatorname{Pr}$ & 13.0 & 17.4 & 16.3 & 14.6 & 18.3 & 29 & 57 & 30 & 54 \\
\hline $\mathrm{Nd}$ & 45 & 60 & 56 & 45 & 71 & 107 & 185 & 100 & 186 \\
\hline $\mathrm{Sm}$ & 8.4 & 10.9 & 10.4 & 5.8 & 13.3 & 18.7 & 26 & 16.1 & 26 \\
\hline $\mathrm{Eu}$ & 1.3 & 1.2 & 1.2 & 1.3 & 3.5 & 3.4 & 2.3 & 1.3 & 5.0 \\
\hline $\mathrm{Gd}$ & 7.6 & 10.1 & 8.8 & 3.5 & 9.5 & 15.8 & 17.2 & 13.4 & 19.9 \\
\hline $\mathrm{Tb}$ & 1.4 & 1.4 & 1.5 & 0.53 & 1.5 & 2.3 & 2.0 & 1.6 & 2.2 \\
\hline Dy & 7.0 & 8.4 & 8.8 & 2.5 & 7.5 & 11.5 & 9.0 & 8.5 & 10.0 \\
\hline Ho & 1.5 & 1.9 & 2.0 & 0.43 & 1.5 & 2.4 & 1.7 & 1.5 & 2.1 \\
\hline $\mathrm{Er}$ & 4.2 & 5.2 & 5.9 & 0.82 & 3.7 & 6.3 & 3.7 & 3.0 & 6.0 \\
\hline $\mathrm{Tm}$ & 0.57 & 0.83 & 0.94 & 0.10 & 0.51 & 0.83 & 0.52 & 0.41 & 0.81 \\
\hline $\mathrm{Yb}$ & 3.7 & 5.8 & 6.5 & 0.56 & 3.4 & 5.4 & 4.2 & 2.4 & 4.9 \\
\hline $\mathrm{Lu}$ & 0.59 & 0.91 & 1.1 & 0.08 & 0.45 & 0.84 & 0.71 & 0.37 & 0.69 \\
\hline$[\mathrm{La} / \mathrm{Yb}]_{n}$ & 10 & 7 & 6 & 72 & 12 & 11 & 36 & 31 & 27 \\
\hline $\mathrm{Eu} / \mathrm{Eu}^{*}$ & 0.49 & 0.35 & 0.39 & 0.83 & 0.90 & 0.59 & 0.31 & 0.26 & 0.64 \\
\hline Fe\# & 0.80 & 0.87 & 0.78 & 0.86 & 0.65 & 0.69 & 0.67 & 0.80 & 0.62 \\
\hline Peral (mol\%) & 1.10 & 1.04 & 0.94 & 1.00 & 1.01 & 1.12 & 0.97 & 1.00 & 0.84 \\
\hline $\operatorname{Agp}(\mathrm{mol} \%)$ & 0.77 & 0.89 & 0.90 & 0.81 & 0.83 & 0.69 & 0.86 & 0.85 & 0.66 \\
\hline $\mathrm{Zr}+\mathrm{Nb}+\mathrm{Ce}+\mathrm{Y}$ & 296 & 407 & 374 & 377 & 465 & 798 & 893 & 571 & 1146 \\
\hline $\mathrm{K}_{2} \mathrm{O}+\mathrm{Na}_{2} \mathrm{O} / \mathrm{CaO}$ & 8.1 & 7.3 & 7.7 & 6.4 & 4.6 & 3.0 & 5.5 & 7.4 & 1.2 \\
\hline
\end{tabular}

Location of samples is given in Fig. 1 and Appendix 1. New analyses, except for major elements in sample 24 quoted from McCarthy (1977, p. 247). 

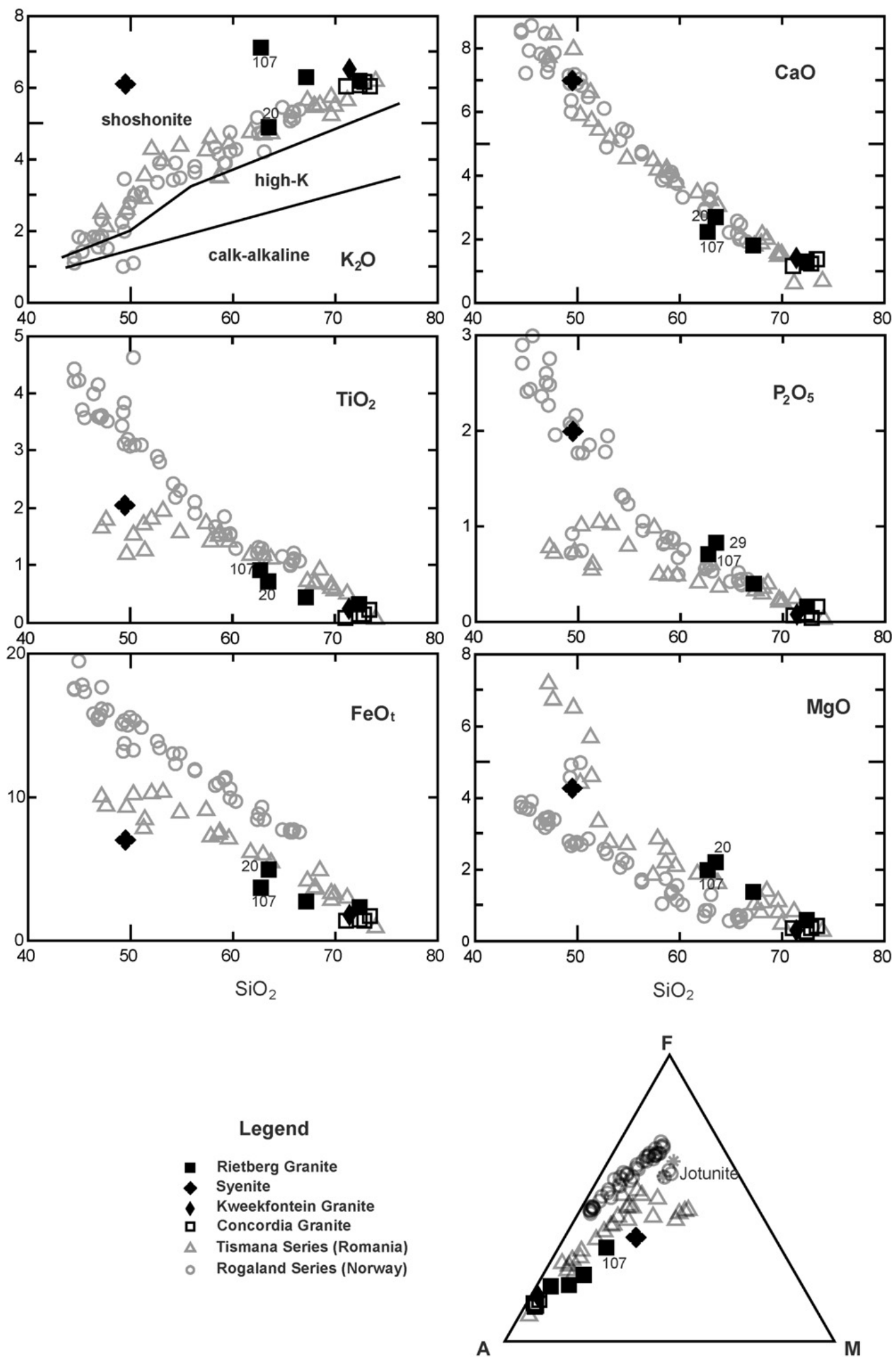

Fig. 3. Concordia, Kweekfontein and Rietberg Granite compositions plotted in Harker diagrams for major elements and in $\mathrm{AFM}\left(\mathrm{Na}_{2} \mathrm{O}+\mathrm{K}_{2} \mathrm{O}\right.$, $\mathrm{FeOt}, \mathrm{MgO}$ ) diagram. On the $\mathrm{K}_{2} \mathrm{O}$ vs. $\mathrm{SiO}_{2}$ diagram, dividers are after Peccerillo and Taylor (1976). The data for the Rogaland and Tismana trends are from Vander Auwera et al. (1998b) and Duchesne et al. (1998), respectively. The position of jotunite is also indicated in the AFM diagram (see text). The numbered samples are particularly discussed in the text. 

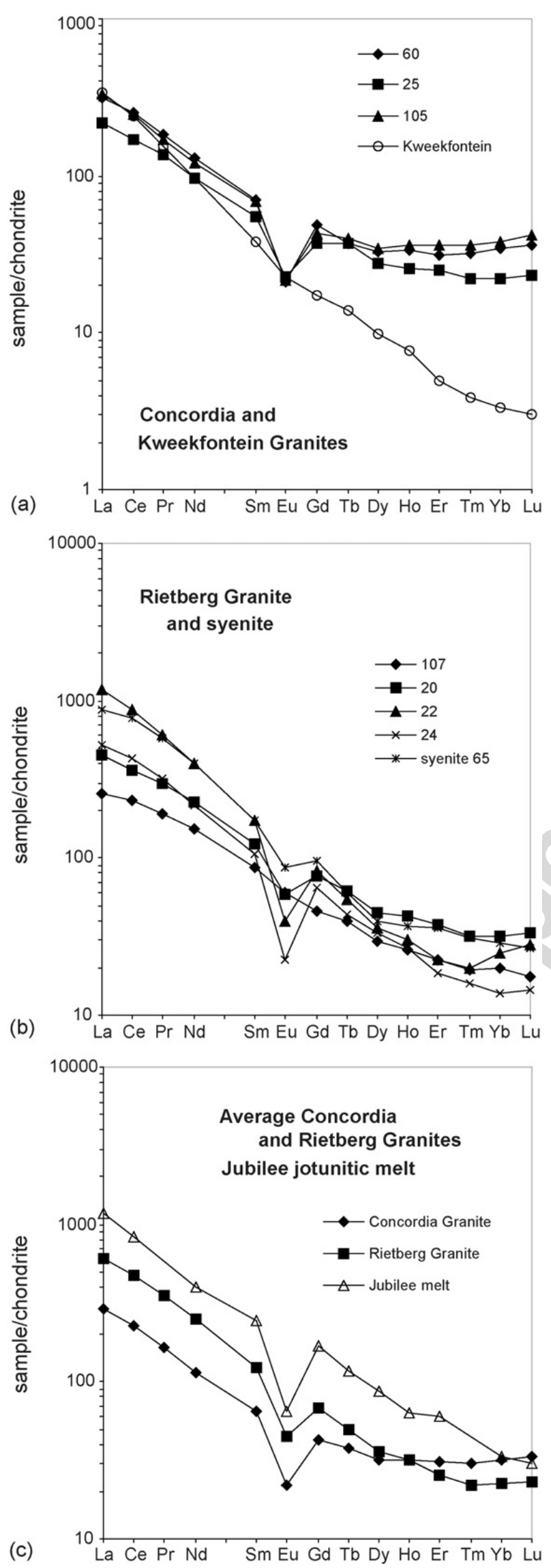

Carpathians) series, which is a typical shoshonitic series (Duchesne et al., 1998) emplaced in a post-collisional environment (Liégeois et al., 1996, 1998). The Rietberg Granite displays a larger range of $\mathrm{SiO}_{2}$ values than the Concordia Granite and shows obvious affinities with the Rogaland and Tismana reference series in $\mathrm{K}_{2} \mathrm{O}, \mathrm{P}_{2} \mathrm{O}_{5}, \mathrm{TiO}_{2}$, and $\mathrm{CaO}$ contents (Fig. 3). With Fe\# $(=\mathrm{FeOt} / \mathrm{FeOt}+\mathrm{MgO})$ of $0.65-0.80$ (Fig. 3), the Rietberg trend is, however, distinctly different from the Rogaland trend (Fe\# ca. 0.91 above $59 \% \mathrm{SiO}_{2}$, Duchesne and Wilmart, 1997), but quite similar to the Tismana trend (Fe\# 0.60-0.80, Duchesne et al., 1998). The Rietberg Granite may, therefore, be viewed as belonging to a Mgrich, potassic series transitional to calc-alkaline trend, as defined for charnockites of the Louis Lake Batholith (Frost et al., 2000).

Finally, the Concordia, Kweekfontein and Rietberg Granites display common overall characteristics and particularly the high $\mathrm{K}_{2} \mathrm{O}$ and trace element contents (Fig. 3), and they show A-type affinities (Whalen et al., 1987), as do also the Rogaland and Tismana trends (Fig. 5a). In the post-collisional granite chemical discrimination diagram of Liégeois et al. (1998) (Fig. 5b), they plot in the shoshonitic field together with the Tismana trend, emphasizing their high contents in $\mathrm{U}$, $\mathrm{Th}$, and $\mathrm{Rb}$.

\subsubsection{Koperberg Suite}

New major and trace element analyses (including REE) of 25 samples from the Koperberg Suite are presented in Table 2 with emphasis on a variety of rock types from different bodies (see Lombaard, 1986), in particular Koperberg Central-Carolusberg West, Jubilee, Wheal Julia, and Homeep East (see Fig. 2 and Appendix 1). Furthermore, trace element analyses are presented for separated plagioclase from 15 samples (Table 3) and for two apatite separates (Table 4). In addition, a new rock type in the Koperberg Suite, called jotunite (=Fe-, Ti-, P-rich hypersthene monzodiorite) (samples 36 and 53), has been identified within the two-pyroxene granulite defined by Clifford et al. (1975) and analysed (Table 5). The major element composition of the two O'okiep is typical of jotunites with high contents of $\mathrm{Fe}$, Ti and P. The REE distributions (Fig. 6) show small negative Eu anomalies, small $[\mathrm{La} / \mathrm{Yb}]_{n}$ ratios $(c a .6)$ and chondrite-normalized La values between 200 and 300 .

Fig. 4. Chondrite-normalized REE distribution in the O'okiep granites and syenite: (a) Concordia and Kweekfontein Granites; (b) Rietberg Granite samples and syenite 65; (c) comparison between average Concordia and Rietberg Granites and Jubilee inverted jotunitic melt. 
Table 2

Whole-rock compositions of the various lithologies from the Koperberg Suite

\begin{tabular}{|c|c|c|c|c|c|c|c|c|c|c|c|c|c|c|}
\hline & \multicolumn{3}{|c|}{ Anorthosites } & \multirow{2}{*}{$\begin{array}{l}\text { Tonalite } \\
\text { Sample } \\
30^{\mathrm{a}}\end{array}$} & \multicolumn{3}{|c|}{ Leuconorite } & \multicolumn{4}{|l|}{ Norite } & \multicolumn{3}{|c|}{ Melanorite } \\
\hline & $\begin{array}{l}\text { Sample } \\
70^{\mathrm{b}}\end{array}$ & $\begin{array}{l}\text { Sample } \\
66\end{array}$ & $\begin{array}{l}\text { Sample } \\
108\end{array}$ & & $\begin{array}{l}\text { Sample } \\
119\end{array}$ & $\begin{array}{l}\text { Sample } \\
120\end{array}$ & $\begin{array}{l}\text { Sample } \\
121\end{array}$ & $\begin{array}{l}\text { Sample } \\
85^{\mathrm{b}}\end{array}$ & $\begin{array}{l}\text { Sample } \\
86^{\mathrm{b}}\end{array}$ & $\begin{array}{l}\text { Sample } \\
116\end{array}$ & $\begin{array}{l}\text { Sample } \\
122\end{array}$ & $\begin{array}{l}\text { Sample } \\
88^{\mathrm{b}}\end{array}$ & $\begin{array}{l}\text { Sample } \\
87^{\mathrm{b}}\end{array}$ & $\begin{array}{l}\text { Sample } \\
110\end{array}$ \\
\hline \multicolumn{15}{|c|}{ Major elements (\%) } \\
\hline $\mathrm{SiO}_{2}$ & 55.17 & 55.70 & 54.66 & 65.63 & 50.79 & 51.72 & 52.09 & 50.30 & 48.99 & 48.01 & 52.52 & 47.51 & 44.25 & 41.25 \\
\hline $\mathrm{TiO}_{2}$ & 0.05 & 0.09 & 0.13 & 0.03 & 0.35 & 0.54 & 0.53 & 0.36 & 0.31 & 1.38 & 0.34 & 0.60 & 1.55 & 0.79 \\
\hline $\mathrm{Al}_{2} \mathrm{O}_{3}$ & 26.70 & 25.76 & 24.08 & 21.12 & 21.65 & 23.19 & 22.22 & 16.34 & 14.80 & 18.61 & 16.94 & 5.54 & 7.69 & 8.31 \\
\hline $\mathrm{Fe}_{2} \mathrm{O}_{3} \mathrm{t}$ & 1.42 & 1.25 & 3.77 & 0.46 & 11.01 & 9.02 & 6.92 & 14.08 & 15.49 & 12.30 & 10.25 & 22.13 & 21.93 & 30.52 \\
\hline $\mathrm{MnO}$ & 0.02 & 0.02 & 0.04 & 0.07 & 0.08 & 0.07 & 0.05 & 0.20 & 0.29 & 0.14 & 0.15 & 0.30 & 0.29 & 0.39 \\
\hline $\mathrm{MgO}$ & 0.41 & 0.66 & 0.98 & 0.15 & 3.70 & 2.58 & 1.91 & 8.04 & 9.13 & 5.26 & 7.69 & 16.70 & 14.82 & 12.09 \\
\hline $\mathrm{CaO}$ & 9.84 & 8.37 & 8.62 & 6.08 & 6.92 & 7.98 & 9.25 & 5.92 & 5.11 & 7.74 & 6.32 & 1.66 & 3.86 & 2.45 \\
\hline $\mathrm{Na}_{2} \mathrm{O}$ & 4.14 & 5.71 & 4.65 & 5.36 & 3.75 & 4.29 & 4.54 & 3.16 & 2.57 & 3.33 & 2.52 & 0.96 & 1.16 & 0.75 \\
\hline $\mathrm{K}_{2} \mathrm{O}$ & 0.92 & 0.85 & 0.81 & 1.11 & 0.94 & 0.71 & 1.04 & 0.55 & 0.63 & 0.43 & 0.95 & 0.57 & 0.20 & 0.30 \\
\hline $\mathrm{P}_{2} \mathrm{O}_{5}$ & 0.23 & 0.23 & 0.01 & 0.12 & 0.08 & 0.01 & 0.14 & 0.08 & 0.07 & 1.05 & 0.13 & 0.07 & 0.68 & 0.11 \\
\hline LOI & 0.22 & 0.47 & 1.44 & 0.25 & 1.05 & 0.06 & 0.97 & 0.81 & 1.18 & 0.84 & 0.81 & 1.19 & 1.84 & \\
\hline Total $^{\mathrm{c}}$ & 99.16 & 99.31 & 99.22 & 100.39 & 100.40 & 100.60 & 99.73 & 101.03 & 98.71 & 100.04 & 98.77 & 100.63 & 100.61 & 99.27 \\
\hline \multicolumn{15}{|c|}{ Trace elements (ppm) } \\
\hline $\mathrm{U}$ & 0.9 & 0.6 & 0.7 & 1.1 & 0.4 & 0.3 & 1.0 & 0.1 & 0.9 & 2.3 & 0.9 & 0.3 & 0.5 & 2.7 \\
\hline $\mathrm{Th}$ & 16.1 & 4.2 & 12.5 & 7.3 & 2.3 & & 4.2 & 0.4 & 1.6 & 21.3 & 4.0 & 1.8 & 3.0 & 29.2 \\
\hline $\mathrm{Zr}$ & 135 & 52 & 71 & 77 & 25 & 19 & 185 & 16 & 103 & 795 & 149 & 34 & 71 & 226 \\
\hline $\mathrm{Hf}$ & 4.5 & 1.2 & 2.4 & 2.2 & & & 3.9 & 0.6 & 2.0 & 24 & 3.9 & 1.1 & 1.8 & 8.3 \\
\hline $\mathrm{Nb}$ & 1.9 & 3.5 & 2.9 & 0.5 & 4.1 & 8.2 & 7.2 & 1.5 & 3.0 & 15 & 3.4 & 1.8 & 11 & 5.1 \\
\hline $\mathrm{Ta}$ & 0.23 & 0.31 & 0.12 & 0.03 & 0.36 & 0.33 & 0.51 & 0.18 & 0.24 & 1.00 & 0.30 & 0.32 & 0.82 & 0.29 \\
\hline $\mathrm{Rb}$ & 8.7 & 8.4 & 9.0 & 35 & 35 & 7.91 & 36 & 8.3 & 14.0 & 13.5 & 36 & 18.7 & 4.1 & 10.4 \\
\hline Cs & 0.2 & 0.2 & 0.4 & 0.7 & 0.8 & 0.3 & 1.1 & 0.5 & 0.8 & 0.5 & 1.1 & 0.5 & 0.2 & 0.6 \\
\hline $\mathrm{Sr}$ & 1206 & 672 & 707 & 455 & 871 & 951 & 892 & 625 & 314 & 552 & 639 & 44 & 168 & 242 \\
\hline $\mathrm{Ba}$ & 329 & 400 & 361 & 160 & 434 & 281 & 257 & 178 & 126 & 288 & 163 & 173 & 75 & 56 \\
\hline $\mathrm{V}$ & 17 & 41 & 86 & 5.0 & 308 & 318 & 212 & 323 & 219 & 306 & 89 & 290 & 423 & 617 \\
\hline $\mathrm{Cr}$ & 110 & 27 & 12.0 & 4.6 & 22 & 26 & 50 & 555 & 438 & 413 & 508 & 2769 & 2265 & 93 \\
\hline Co & 1.6 & 3.6 & 6.7 & 0.5 & 32 & 28 & 20 & 50 & 54 & 42 & 42 & 98 & 96 & 85 \\
\hline $\mathrm{Zn}$ & 13 & 34 & 31 & 76 & 147 & 130 & 71 & 215 & 288 & 193 & 206 & 349 & 384 & 503 \\
\hline $\mathrm{Ni}$ & 351 & 19 & 8 & 0 & 78 & 46 & 64 & 262 & 236 & 147 & 180 & 883 & 757 & 160 \\
\hline $\mathrm{Cu}$ & 460 & 1994 & 272 & 88 & 815 & 4296 & 667 & 11905 & 1403 & 9502 & 1503 & 33976 & 23449 & 23155 \\
\hline $\mathrm{Ga}$ & 28 & 31 & 28 & 24 & 32 & 33 & 31 & 30 & 27 & 30 & 27 & 24 & 23 & 33 \\
\hline $\mathrm{Pb}$ & 28 & 23 & 28 & 26 & 15 & 25 & 18 & 12 & 14 & 14 & 15 & 7 & 6 & 12 \\
\hline $\mathrm{Y}$ & 13.0 & 20 & 8.2 & 5.6 & 8.8 & 10.4 & 21 & 10.8 & 24 & 55 & 27 & 24 & 42 & 37 \\
\hline $\mathrm{La}$ & & 63 & & 43 & 27 & 43 & 59 & 24 & 28 & 93 & 57 & 13.5 & 32 & 44 \\
\hline $\mathrm{Ce}$ & 123 & 98 & 98 & 73 & 43 & 52 & 95 & 32 & 49 & 214 & 77 & 22 & 75 & 74 \\
\hline $\operatorname{Pr}$ & & 11.4 & & 7.0 & 5.2 & & & 2.2 & 5.3 & 30 & 9.5 & 3.3 & 10.9 & 8.7 \\
\hline $\mathrm{Nd}$ & 46 & 41 & 32 & 22 & 14.6 & 17.3 & 35 & 10.3 & 18.8 & 119 & 35 & 14.1 & 43 & 30 \\
\hline $\mathrm{Sm}$ & 7.3 & 6.6 & 4.0 & 3.4 & 2.4 & 1.8 & 5.6 & 1.6 & 3.5 & 20.5 & 5.5 & 2.0 & 8.5 & 5.2 \\
\hline $\mathrm{Eu}$ & 2.1 & 2.1 & 2.4 & 1.9 & 1.1 & 1.4 & 1.5 & 1.1 & 1.3 & 2.9 & 1.3 & 0.51 & 1.2 & 0.74 \\
\hline $\mathrm{Gd}$ & 4.4 & 4.1 & 2.1 & 2.3 & 0.9 & 0.8 & 4.1 & 1.4 & 3.1 & 18.0 & 4.2 & 1.8 & 7.1 & 4.6 \\
\hline $\mathrm{Tb}$ & & 0.66 & & 0.28 & & & & 0.21 & 0.47 & 2.8 & 0.80 & & 1.2 & 0.76 \\
\hline Dy & 2.8 & 3.5 & & 1.3 & 1.1 & 1.2 & 3.2 & 1.5 & 2.8 & 11.0 & 4.2 & 3.0 & 6.7 & 4.7 \\
\hline Ho & & & $>$ & 0.24 & & & & & & 2.2 & 0.87 & & & \\
\hline $\mathrm{Er}$ & 1.4 & 1.7 & 0.98 & 0.58 & 0.59 & 1.3 & 2.6 & 0.95 & 2.5 & 5.6 & 2.8 & 2.7 & 4.1 & 4.9 \\
\hline $\mathrm{Tm}$ & & 0.22 & & 0.10 & & & & 0.16 & 0.44 & 0.69 & 0.41 & 0.47 & 0.60 & 0.99 \\
\hline $\mathrm{Yb}$ & 0.97 & 1.2 & 0.90 & 0.55 & 0.70 & 1.7 & 2.8 & 1.7 & 3.8 & 4.6 & 2.8 & 3.3 & 4.1 & 8.9 \\
\hline $\mathrm{Lu}$ & 0.10 & 0.21 & 0.13 & 0.10 & 0.15 & 0.23 & 0.39 & 0.30 & 0.63 & 0.75 & 0.47 & 0.54 & 0.63 & 1.61 \\
\hline$[\mathrm{Ce} / \mathrm{Yb}]_{n}$ & 35 & 23 & 30 & 37 & 17 & 9 & 9 & 5 & 4 & 13 & 8 & 2 & 5 & 2 \\
\hline $\mathrm{Eu} / \mathrm{Eu}^{*}$ & 1.07 & 1.17 & 2.33 & 1.97 & 1.84 & 3.13 & 0.93 & 2.20 & 1.19 & 0.46 & 0.77 & 0.80 & 0.46 & 0.45 \\
\hline
\end{tabular}


Table 2 (Continued)

\begin{tabular}{|c|c|c|c|c|c|c|c|c|c|c|c|}
\hline & \multicolumn{2}{|c|}{ Hypersthenite } & \multirow{2}{*}{$\begin{array}{l}\text { Glimmerite } \\
\text { Sample } \\
123\end{array}$} & \multicolumn{2}{|c|}{ Magnetitite } & \multicolumn{6}{|c|}{ Biotite diorite } \\
\hline & $\begin{array}{l}\text { Sample } \\
90^{\mathrm{b}}\end{array}$ & $\begin{array}{l}\text { Sample } \\
117\end{array}$ & & $\begin{array}{l}\text { Sample } \\
82^{\mathrm{b}}\end{array}$ & $\begin{array}{l}\text { Sample } \\
125^{\mathrm{b}}\end{array}$ & $\begin{array}{l}\text { Sample } \\
78^{\mathrm{b}}\end{array}$ & $\begin{array}{l}\text { Sample } \\
112\end{array}$ & $\begin{array}{l}\text { Sample } \\
109\end{array}$ & $\begin{array}{l}\text { Sample } \\
114\end{array}$ & $\begin{array}{l}\text { Sample } \\
118\end{array}$ & $\begin{array}{l}\text { Sample } \\
126\end{array}$ \\
\hline \multicolumn{12}{|c|}{ Major elements (\%) } \\
\hline $\mathrm{SiO}_{2}$ & 48.30 & 41.50 & 40.50 & 25.87 & 29.38 & 50.97 & 49.38 & 54.00 & 60.01 & 43.74 & 52.28 \\
\hline $\mathrm{TiO}_{2}$ & 0.55 & 0.95 & 5.00 & 7.11 & 1.89 & 0.93 & 1.00 & 0.74 & 0.96 & 2.41 & 0.26 \\
\hline $\mathrm{Al}_{2} \mathrm{O}_{3}$ & 4.10 & 2.90 & 11.00 & 2.45 & 2.69 & 22.63 & 22.37 & 22.11 & 16.82 & 19.43 & 21.78 \\
\hline $\mathrm{Fe}_{2} \mathrm{O}_{3} \mathrm{t}$ & 24.33 & 25.90 & 10.80 & 47.91 & 39.66 & 10.03 & 8.80 & 5.80 & 8.14 & 8.94 & 7.35 \\
\hline $\mathrm{MnO}$ & 0.49 & 0.25 & 0.03 & 0.37 & 0.43 & 0.08 & 0.06 & 0.08 & 0.11 & 0.16 & 0.13 \\
\hline $\mathrm{MgO}$ & 20.41 & 19.00 & 17.50 & 11.48 & 11.39 & 2.67 & 3.11 & 1.55 & 2.32 & 7.11 & 4.83 \\
\hline $\mathrm{CaO}$ & 1.21 & 3.80 & 3.50 & 2.91 & 6.62 & 6.68 & 7.09 & 5.47 & 6.55 & 6.51 & 7.95 \\
\hline $\mathrm{Na}_{2} \mathrm{O}$ & 0.00 & 0.35 & 0.50 & 0.06 & 0.03 & 4.06 & 4.18 & 5.10 & 3.42 & 3.95 & 3.82 \\
\hline $\mathrm{K}_{2} \mathrm{O}$ & 0.00 & 2.00 & 6.70 & 0.09 & 0.07 & 1.80 & 1.85 & 2.72 & 0.72 & 4.30 & 0.71 \\
\hline $\mathrm{P}_{2} \mathrm{O}_{5}$ & 0.09 & 1.50 & 1.90 & 2.05 & 4.80 & 0.05 & 0.43 & 0.33 & 0.31 & 1.33 & 0.23 \\
\hline LOI & 0.00 & 0.81 & 3.27 & 0.00 & 1.51 & 1.26 & 1.88 & 1.66 & 0.86 & 2.00 & 1.38 \\
\hline Total $^{\mathrm{c}}$ & 101.25 & 98.96 & 100.70 & 100.60 & 99.93 & 101.48 & 100.19 & 99.60 & 100.22 & 100.07 & 100.85 \\
\hline \multicolumn{12}{|c|}{ Trace elements (ppm) } \\
\hline $\mathrm{U}$ & 0.2 & 0.3 & 1.2 & 2.1 & 3.0 & 1.0 & 7.9 & 5.9 & 2.7 & 0.2 & 0.4 \\
\hline Th & 0.7 & 2.4 & 4.0 & 8.0 & 16.1 & 5.8 & 418 & 53.1 & 8.8 & 3.2 & 1.5 \\
\hline $\mathrm{Zr}$ & 105 & 55 & 39 & 1260 & 36 & 259 & 1173 & 193 & 666 & 65 & 224 \\
\hline Hf & 2.6 & 1.1 & 0.7 & 37 & 1.0 & 8.5 & 31 & 7.1 & 21 & 1.6 & 6.2 \\
\hline $\mathrm{Nb}$ & 3.9 & 5.5 & 32 & 59 & 4.2 & 5 & 17 & 54 & 15 & 15 & 3 \\
\hline $\mathrm{Ta}$ & 0.25 & 0.46 & 0.71 & 3.08 & 0.44 & 0.55 & 1.1 & 3.3 & 1.3 & 0.58 & 0.15 \\
\hline $\mathrm{Rb}$ & 10.5 & 4.6 & 484 & 5.5 & 4.5 & 6.3 & 132 & 160 & 57 & 206 & 10 \\
\hline Cs & 0.4 & 0.2 & 2.8 & 0.7 & 0.3 & 0.3 & 2.0 & 4.7 & 1.3 & 1.0 & 0.0 \\
\hline $\mathrm{Sr}$ & 139 & 132 & 136 & 64 & 18 & 753 & 786 & 286 & 303 & 882 & 700 \\
\hline $\mathrm{Ba}$ & 94 & 132 & 5716 & 219 & 16 & 176 & 725 & 294 & 95 & 4844 & 199 \\
\hline $\mathrm{V}$ & 228 & 324 & 591 & 1130 & 1265 & 436 & 200 & 79 & 112 & 337 & 85 \\
\hline $\mathrm{Cr}$ & 2213 & 3508 & 2383 & 239 & 1023 & 123 & 5 & 33 & 13 & 161 & 189 \\
\hline $\mathrm{Co}$ & 99 & 118 & 121 & 145 & 81 & 32 & 24 & 9 & 14 & 45 & 26 \\
\hline $\mathrm{Zn}$ & 419 & 397 & 332 & 680 & 342 & 204 & 94 & 89 & 89 & 139 & 100 \\
\hline $\mathrm{Ni}$ & 549 & 846 & 945 & 568 & 480 & 115 & 94 & 10 & 10 & 155 & 78 \\
\hline $\mathrm{Cu}$ & 17715 & 57546 & 23543 & 3018 & 14609 & 3285 & 404 & 393 & 40 & 1924 & 1332 \\
\hline $\mathrm{Ga}$ & 21 & 19 & 28 & 40 & 47 & 36 & 39 & 30 & 26 & 27 & 28 \\
\hline $\mathrm{Pb}$ & 6 & 2 & 7 & & 6 & 18 & 33 & 54 & 17 & 18 & 13 \\
\hline $\mathrm{Y}$ & 22 & 33 & 111 & 70 & 195 & 21 & 65 & 116 & 56 & 23 & 16 \\
\hline $\mathrm{La}$ & 14.7 & 54 & 101 & 103 & 181 & 52 & 605 & 92 & 70 & 96 & 39 \\
\hline $\mathrm{Ce}$ & 27 & 118 & 286 & 266 & 481 & 88 & 1091 & 148 & 149 & 211 & 72 \\
\hline $\operatorname{Pr}$ & 3.5 & & & & 69.6 & 10.7 & 114 & & 19.8 & & 8.1 \\
\hline $\mathrm{Nd}$ & 13.6 & 65 & 172 & 146 & 294 & 35 & 379 & 65 & 75 & 100 & 30 \\
\hline $\mathrm{Sm}$ & 2.7 & 10.7 & 30 & 25.7 & 60 & 5.7 & 53 & 14.0 & 13.8 & 14.9 & 5.2 \\
\hline $\mathrm{Eu}$ & 0.68 & 1.7 & 4.1 & 3.0 & 3.0 & 1.4 & 3.8 & 2.5 & 2.5 & 3.0 & 1.1 \\
\hline $\mathrm{Gd}$ & 2.4 & 7.4 & 22 & 19.1 & 61 & 2.9 & 29 & 15.0 & 12.1 & 9.7 & 4.2 \\
\hline $\mathrm{Tb}$ & & & & & 7.8 & 0.63 & & & 2.03 & & 0.56 \\
\hline Dy & 3.1 & 5.2 & 15.2 & 11.9 & 36.9 & 3.6 & 14.1 & 18.2 & 10.7 & 5.4 & 2.8 \\
\hline Ho & & & & & 7.3 & 0.67 & 2.5 & & 2.2 & & 0.66 \\
\hline $\mathrm{Er}$ & 2.3 & 3.3 & 11.2 & 7.0 & 17.4 & 2.4 & 5.1 & 12.6 & 6.2 & 2.3 & 1.6 \\
\hline $\mathrm{Tm}$ & 0.42 & & & & 2.1 & 0.38 & 0.69 & & 0.90 & & 0.24 \\
\hline $\mathrm{Yb}$ & 3.1 & 2.7 & 9.4 & 6.1 & 12.9 & 2.7 & 3.9 & 11.9 & 5.5 & 1.3 & 1.6 \\
\hline $\mathrm{Lu}$ & 0.50 & 0.38 & 1.4 & 1.0 & 1.8 & 0.49 & 0.59 & 1.74 & 0.91 & 0.15 & 0.26 \\
\hline$[\mathrm{Ce} / \mathrm{Yb}]_{n}$ & 2 & 12 & 8 & 12 & 10 & 9 & 77 & 3 & 8 & 45 & 12 \\
\hline $\mathrm{Eu} / \mathrm{Eu}^{*}$ & 0.80 & 0.55 & 0.46 & 0.39 & 0.15 & 0.95 & 0.27 & 0.52 & 0.58 & 0.71 & 0.69 \\
\hline
\end{tabular}

New analyses, except for major elements in.

a Sample 30 quoted from Clifford et al. (1975, p.166).

b Tom Clifford unpublished data (1984) samples 78, 85 and 90 (analyst P. Richardson) and (1985) samples 70, 82, 86 and 125 (analyst S. Hall).

c Total includes $\mathrm{Cu}$. Location of samples is given in Fig. 2 and Appendix 1. 
Table 3

Plagioclase composition from cumulate rocks and biotite diorite from the Koperberg Suite

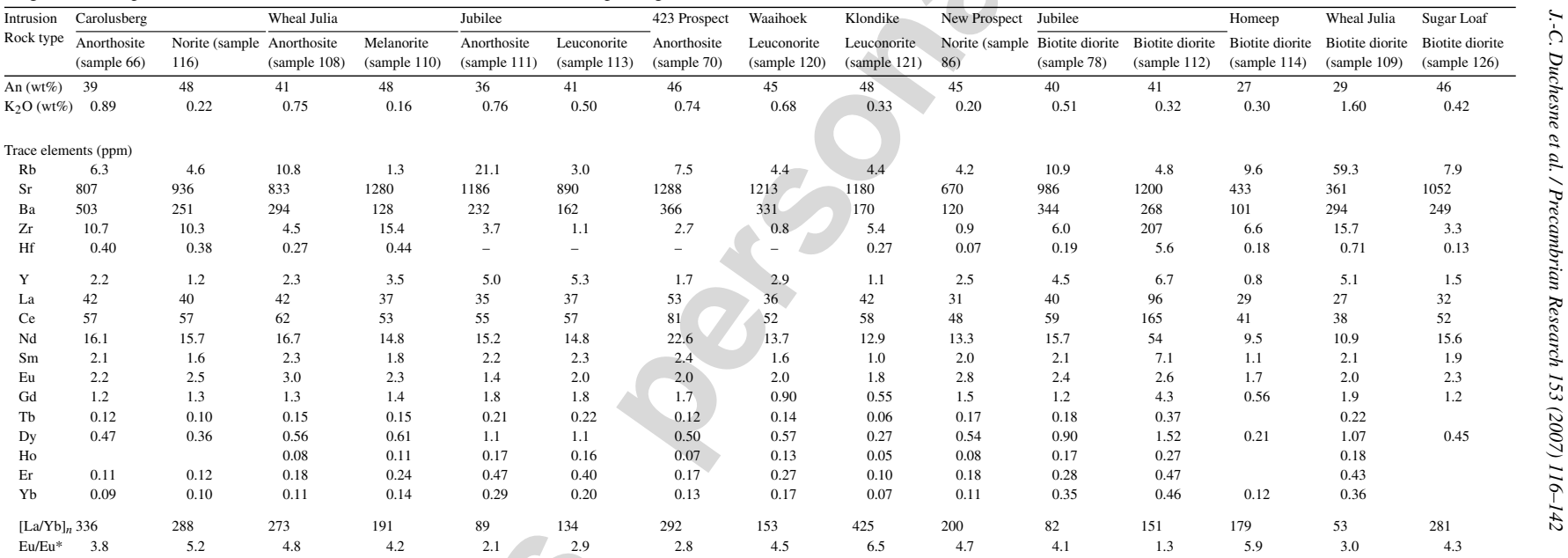




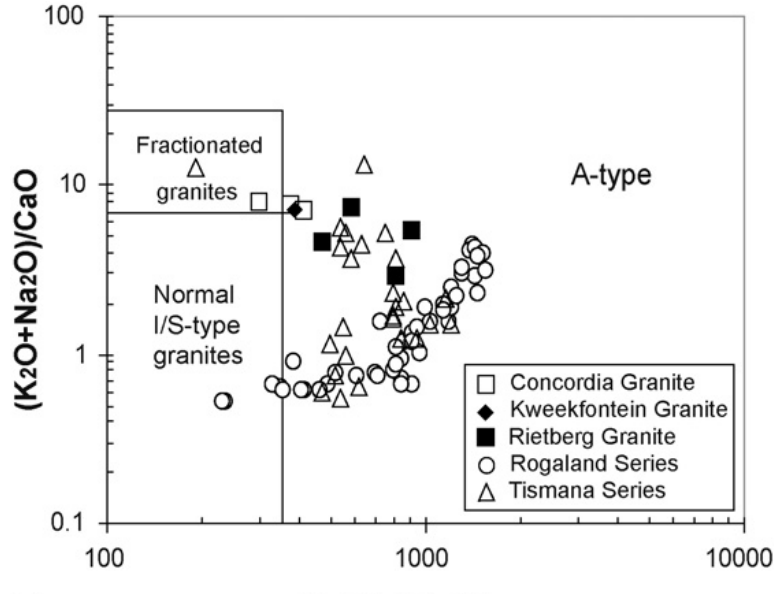

(a)

$\mathrm{Zr}+\mathrm{Nb}+\mathrm{Ce}+\mathrm{Y}$

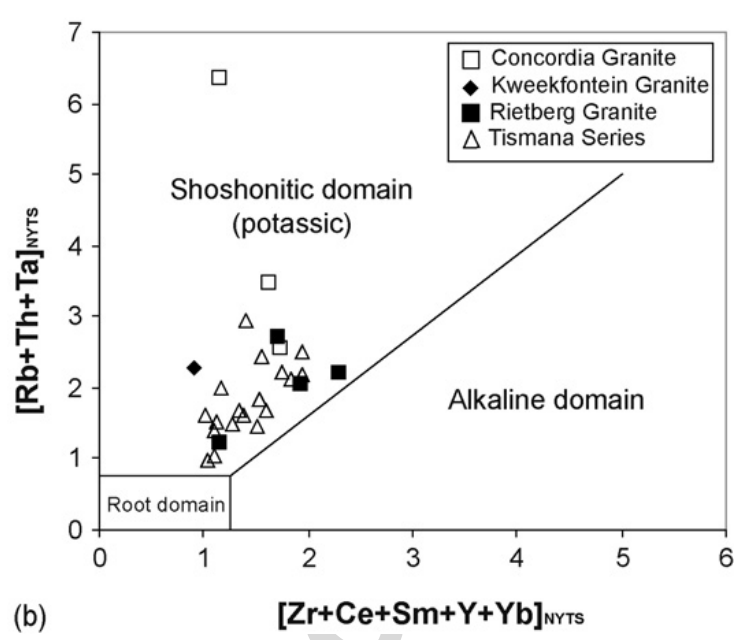

Fig. 5. O'okiep granite compositions plotted in trace element discrimination diagrams. (a) $\mathrm{Zr}+\mathrm{Nb}+\mathrm{Ce}+\mathrm{Y}$ vs. $\mathrm{K}_{2} \mathrm{O}+\mathrm{Na}_{2} \mathrm{O} / \mathrm{CaO}$ diagram of Whalen et al. (1987). Granitic rocks are compared to the Rogaland and Tismana series. (b) Synthetic diagram with sliding normalization of Liégeois et al. (1998). NYTS means that each sample is normalized to the interpolated virtual rock of the Yenchichi-Telabit reference magmatic series that has the same $\mathrm{SiO}_{2}$ content (see Liégeois et al., 1998).

The jotunite samples are typical igneous rocks and they consist of a granular aggregate of antiperthitic andesine, clinopyroxene, poikilitic hypersthene, ilmenite and trellis-textured Ti-magnetite. Spherical pyrrhotite and

Table 4

Trace element contents of separated apatites from the Koperberg Suite

\begin{tabular}{|c|c|c|c|}
\hline & $\begin{array}{l}\text { Norite } \\
\text { (sample 116) }\end{array}$ & $\begin{array}{l}\text { Magnetitite } \\
\text { (sample 115) }\end{array}$ & $\begin{array}{l}\text { Diorite (sample } \\
\text { Ho- } 54^{\text {a }} \text { ) }\end{array}$ \\
\hline \multicolumn{4}{|c|}{ Trace elements (ppm) } \\
\hline $\mathrm{La}$ & 580 & 811 & 617 \\
\hline $\mathrm{Ce}$ & 2376 & 3509 & 2320 \\
\hline $\operatorname{Pr}$ & 428 & 711 & \\
\hline $\mathrm{Nd}$ & 2211 & 3804 & 2021 \\
\hline $\mathrm{Sm}$ & 545 & 1141 & 499 \\
\hline $\mathrm{Eu}$ & 54 & 59 & 47.95 \\
\hline $\mathrm{Gd}$ & 464 & 1334 & 433 \\
\hline $\mathrm{Tb}$ & 60 & 224 & \\
\hline Dy & 316 & 1389 & 336 \\
\hline Но & 56 & 270 & \\
\hline $\mathrm{Er}$ & 152 & 805 & 152 \\
\hline $\mathrm{Yb}$ & 96 & 545 & 120 \\
\hline $\mathrm{Lu}$ & & & 17.8 \\
\hline$[\mathrm{La} / \mathrm{Yb}]_{n}$ & 3.9 & 1.0 & 3.3 \\
\hline $\mathrm{Eu} / \mathrm{Eu}^{*}$ & 0.32 & 0.15 & 0.31 \\
\hline $\mathrm{Y}$ & 1406 & 6972 & \\
\hline Th & 6.6 & 53.8 & \\
\hline $\mathrm{U}$ & 21.3 & 149 & \\
\hline $\mathrm{Nb}$ & 1.44 & 7.2 & \\
\hline $\mathrm{Ta}$ & 0.6 & 7.0 & \\
\hline $\mathrm{Pb}$ & 4.5 & 10.8 & \\
\hline $\mathrm{Rb}$ & 1.8 & 4.2 & \\
\hline $\mathrm{Sr}$ & 433 & 178 & \\
\hline $\mathrm{Mn}$ & 218 & 341 & \\
\hline
\end{tabular}

Location of samples in Fig. 2 and Appendix 1.

${ }^{a}$ From Conradie and Schoch (1988, p. 414). chalcopyrite grains occur as inclusions in the silicates. Euhedral zircons are common and frequently show inherited cores (Clifford et al., 2004). Apatite is abundant and forms thin elongated prisms, dispersed in all minerals; this character and the granular texture are typical of "chilled" rocks commonly found in anorthosite provinces (Wiebe, 1984; Duchesne et al., 1989) and, in

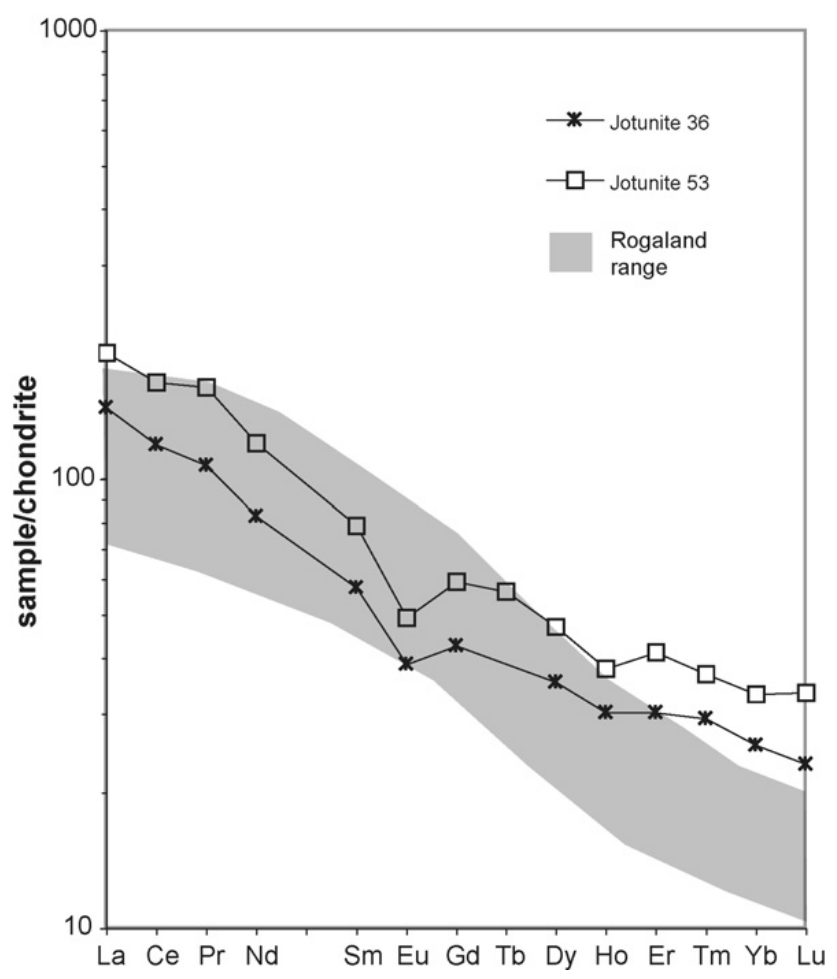

Fig. 6. Chondrite-normalized REE distribution in Koperberg Suite jotunitic rocks (data in Table 5), compared to the range of Rogaland jotunite (Duchesne et al., 1989). 
Table 5

Whole-rock chemical compositions of jotunite from the Koperberg Suite

\begin{tabular}{|c|c|c|}
\hline & Jotunite & \\
\hline & Sample 53 & Sample 36 \\
\hline Major eleme & & \\
\hline $\mathrm{SiO}_{2}$ & 52.29 & 53.43 \\
\hline $\mathrm{TiO}_{2}$ & 2.51 & 2.29 \\
\hline $\mathrm{Al}_{2} \mathrm{O}_{3}$ & 13.28 & 14.42 \\
\hline $\mathrm{Fe}_{2} \mathrm{O}_{3} \mathrm{t}$ & 15.14 & 12.81 \\
\hline $\mathrm{MnO}$ & 0.22 & 0.18 \\
\hline $\mathrm{MgO}$ & 4.36 & 3.87 \\
\hline $\mathrm{CaO}$ & 8.20 & 8.28 \\
\hline $\mathrm{Na}_{2} \mathrm{O}$ & 2.24 & 3.16 \\
\hline $\mathrm{K}_{2} \mathrm{O}$ & 1.50 & 1.48 \\
\hline $\mathrm{P}_{2} \mathrm{O}_{5}$ & 0.96 & 0.50 \\
\hline Total & 100.70 & 100.42 \\
\hline Trace eleme & & \\
\hline S & 1770 & 2010 \\
\hline $\mathrm{U}$ & 0.6 & 0.5 \\
\hline $\mathrm{Th}$ & 1.6 & 1.2 \\
\hline $\mathrm{Zr}$ & 477 & 359 \\
\hline $\mathrm{Hf}$ & 12.5 & 9.3 \\
\hline $\mathrm{Nb}$ & 24 & 19 \\
\hline $\mathrm{Ta}$ & 1.71 & 1.41 \\
\hline $\mathrm{Rb}$ & 46 & 47 \\
\hline Cs & 0.8 & 0.2 \\
\hline $\mathrm{Sr}$ & 186 & 208 \\
\hline $\mathrm{Ba}$ & 970 & 710 \\
\hline V & 320 & 321 \\
\hline $\mathrm{Cr}$ & 114 & 29 \\
\hline Co & 42 & 39 \\
\hline $\mathrm{Zn}$ & 163 & 123 \\
\hline $\mathrm{Ni}$ & 311 & 19 \\
\hline $\mathrm{Cu}$ & 43 & 33 \\
\hline $\mathrm{Ga}$ & 23 & 21 \\
\hline $\mathrm{Pb}$ & 11 & 10 \\
\hline $\mathrm{Y}$ & 83 & 59 \\
\hline $\mathrm{La}$ & 65 & 49 \\
\hline $\mathrm{Ce}$ & 143 & 104 \\
\hline $\operatorname{Pr}$ & 19.4 & 13.0 \\
\hline $\mathrm{Nd}$ & 77 & 53 \\
\hline $\mathrm{Sm}$ & 15.4 & 11.2 \\
\hline $\mathrm{Eu}$ & 3.60 & 2.83 \\
\hline $\mathrm{Gd}$ & 15.40 & 11.10 \\
\hline $\mathrm{Tb}$ & 2.65 & \\
\hline Dy & 14.10 & 10.62 \\
\hline Но & 2.96 & 2.36 \\
\hline $\mathrm{Er}$ & 8.25 & 6.06 \\
\hline $\mathrm{Tm}$ & 1.18 & 0.94 \\
\hline $\mathrm{Yb}$ & 7.33 & 5.63 \\
\hline $\mathrm{Lu}$ & 1.14 & 0.79 \\
\hline$[\mathrm{La} / \mathrm{Yb}]_{n}$ & 6 & 6 \\
\hline $\mathrm{Eu} / \mathrm{Eu}^{*}$ & 0.71 & 0.77 \\
\hline Fe\# & 0.76 & 0.75 \\
\hline
\end{tabular}

Location of samples is given in Fig. 2 and in Appendix 1.
Rogaland, as dyke rocks or as marginal facies to igneous bodies (Demaiffe and Hertogen, 1981; Duchesne and Hertogen, 1988; Robins et al., 1997). The rocks therefore clearly resulted from the crystallization of melts.

Age data for zircons from these jotunites (Clifford et al., 2004) are as follows. In sample 36, zircon magmatic rims gave an age of $1035 \pm 15 \mathrm{Ma}$, and cores yielded well grouped at $1203 \pm 10 \mathrm{Ma}$ ages with some older cores with ages up to $1804 \pm 17 \mathrm{Ma}$; in sample 53, cores show continuous lead loss from $1200 \mathrm{Ma}$ and thin zircon rim ages average at $1069 \pm 44 \mathrm{Ma}$, that is within error similar to the $1035 \pm 15 \mathrm{Ma}$ age, considered to date the jotunite magmatic zircons. These jotunites therefore clearly belong to the same intrusive age event as the Koperberg Suite.

A major issue in the interpretation of whole-rock geochemistry in terms of differentiation processes is to distinguish between cumulates, liquids and crystalladen liquids, and this is an obvious prerequisite to any modelling of the petrogenesis of the rocks. In this work Harker diagrams (Fig. 7) are presented to compare the rock compositions (Table 2) to mineral compositions, and to the well-documented jotunitic liquid line of descent (Vander Auwera et al., 1998b). In Fig. 7, the compositions of minerals, well documented by Conradie and Schoch (1986), Brandriss and Cawthorn (1996) and Van Zwieten et al. (1996), display little variation and may be represented by circles of restricted size.

The anorthosite, leuconorite and norite of the Koperberg Suite show a large variation in composition but plot within the triangle formed by plagioclase, biotite and hypersthene (Fig. 7). As suggested by McIver et al. (1983) and Brandriss and Cawthorn (1996), this reflects the fact that they are mixtures of these minerals and, thus their compositions result from mineral sorting and accumulation processes rather than from direct crystallization of melts. In the same way, the tonalite (sample 30) plots along the plagioclase-quartz tie line and this suggests a cumulate dominated by plagioclase and quartz. Melanorite, hypersthenite and glimmerite are also scattered close to the biotite and hypersthene poles; magnetitites are accumulations of magnetite, ilmenite and apatite in various proportions. None of these rocks represent melts.

Fig. 8 shows the chondrite-normalized REE distribution for the various rock types of the Koperberg Suite. It is obvious that within each group the abundance, the REE distribution, the $\mathrm{La} / \mathrm{Yb}$ ratio and the Eu anomaly vary considerably; no simple distribution appears characteristic of a particular rock type. This is in conflict 

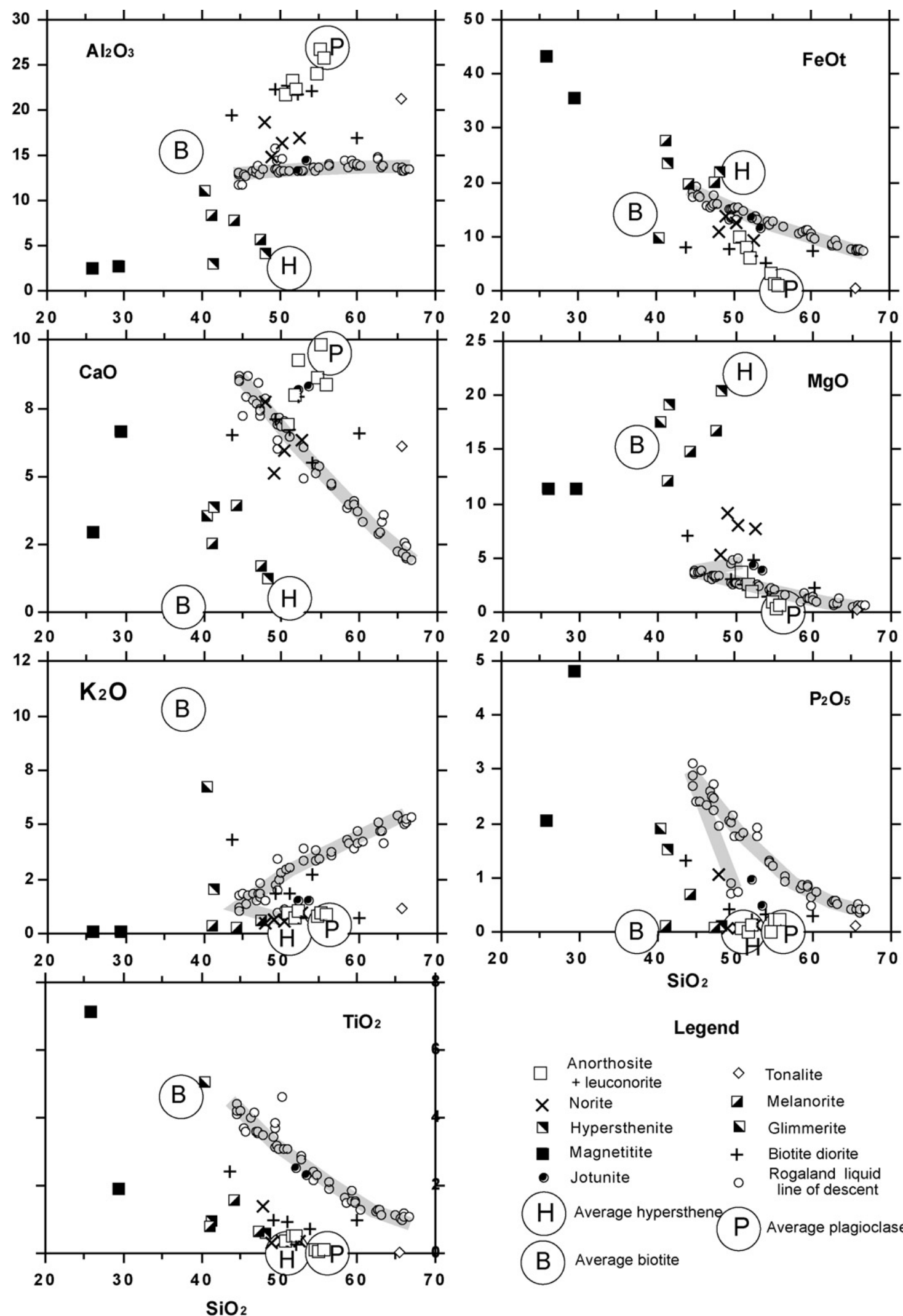

Legend

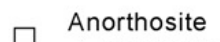

+ leuconorite

$\diamond \quad$ Tonalite

$\times$ Norite

\} \text { Hypersthenite }

- Melanorite

Magnetitite

- Glimmerite

- Jotunite 0 Rogaland liquid

$+\quad$ Biotite diorite

(H) Average hypersthene (P) Average plagioclase

(B) Average biotite

Fig. 7. Whole-rock compositions (Table 2) for the Koperberg Suite plotted in Harker diagrams, together with the Rogaland liquid line of descent (Vander Auwera et al., 1998b). The compositions of the main rock-forming minerals - hypersthene (H), biotite (B) and plagioclase (P) - are contained in the labelled circles (see text). 

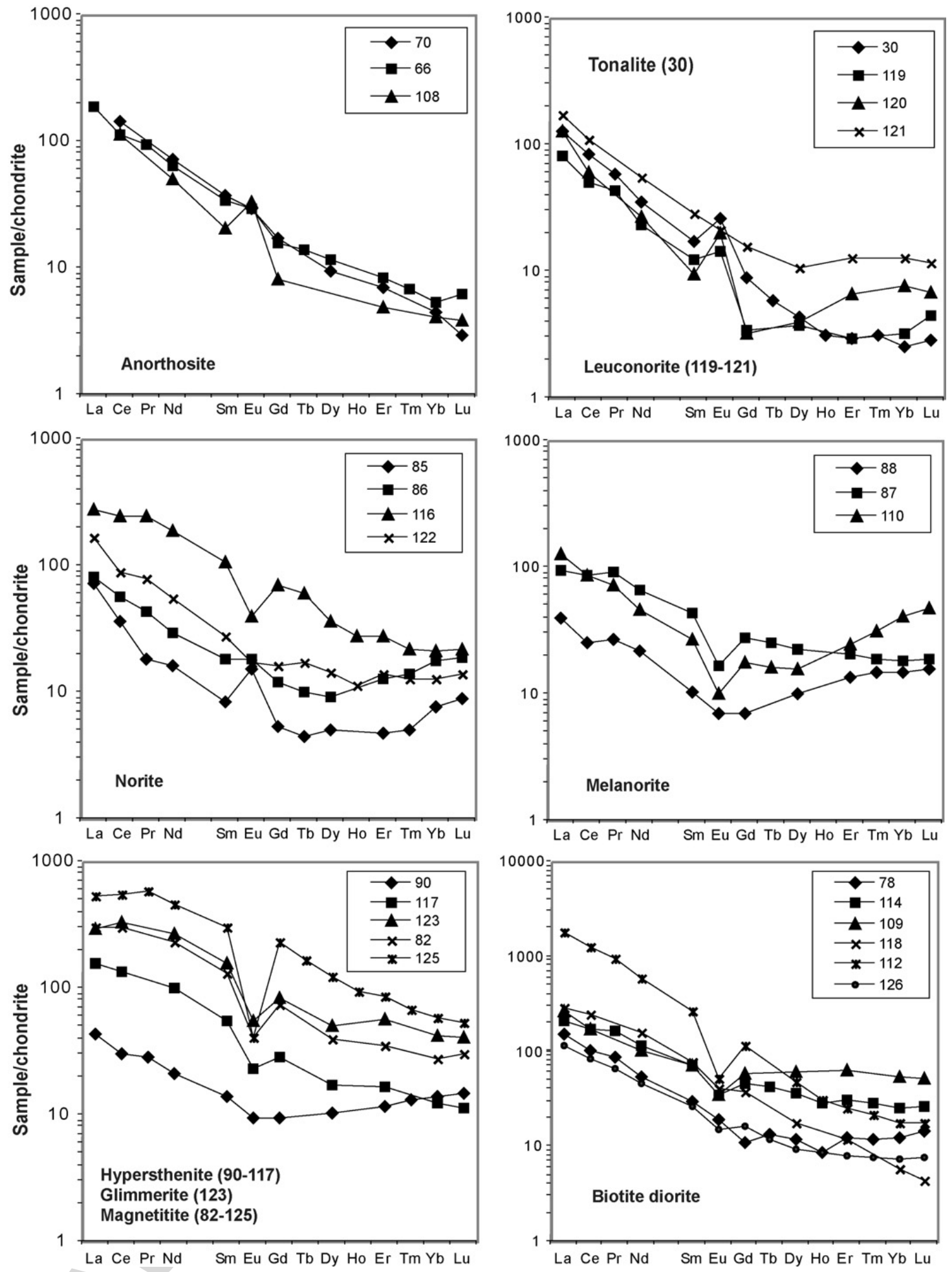

Fig. 8. Chondrite-normalized REE distribution in the various Koperberg lithologies (data in Table 2).

with the views of Conradie and Schoch (1988) who claim that anorthosite is characterized by large positive $\mathrm{Eu}$ anomalies, leuconorite by the absence of Eu anomalies, and norite by negative Eu anomalies. Our data suggest that a classification based on REE alone has little relevance.

\subsection{Isotopes}

$\mathrm{Sr}$ and $\mathrm{Nd}$ isotopic compositions on whole-rocks and plagioclases from the Koperberg Suite in the O'okiep District are reported in Table 6. They complement the data by Clifford et al. (1975, 1995), Brandriss and 


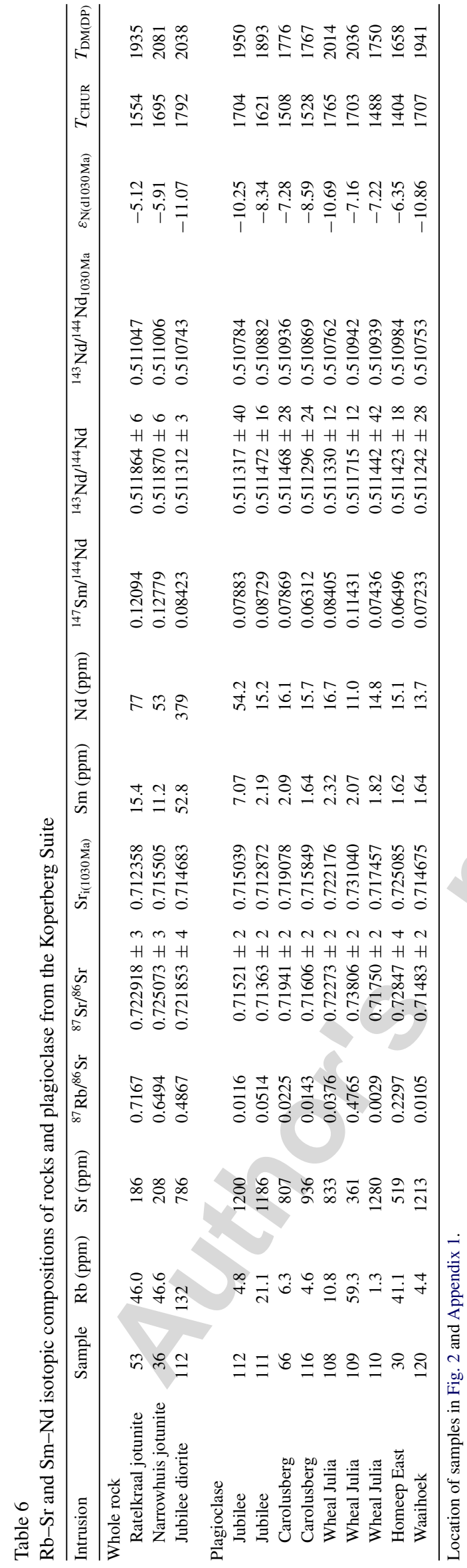

Cawthorn (1996), Van Zwieten et al. (1996) and Geringer et al. (1998) on the O'okiep District.

The new isotopic data (Table 6) together with those already published on the O'okiep District (Fig. 9), confirm the main characteristics previously recognized by Clifford et al. (1995) and Geringer et al. (1998) as follows: (1) the Koperberg rocks show negative $\varepsilon_{\mathrm{Nd}}$ values at $1030 \mathrm{Ma}\left(\varepsilon_{\mathrm{Nd}}(1030 \mathrm{Ma})\right)$ between -5 and -11 of, with an average at $-8.6 \pm 1.6$; (2) the initial $\mathrm{Sr}$ isotope ratios at the age of $1030 \mathrm{Ma}\left(\mathrm{Sr}_{\mathrm{i}}\right)$ spread between 0.709 and 0.748 ; (3) the country rocks (Nababeep gneisses, twopyroxene granulites) and the Concordia Granite also show negative $\varepsilon_{\mathrm{Nd}}(1030 \mathrm{Ma})$ values $(-5$ to -8$)$ and a large interval of variation of $\mathrm{Sr}_{\mathrm{i}}$ from 0.709 to 0.744 . $\mathrm{Nd}$ model ages of the Koperberg Suite are grouped at $T_{\mathrm{CHUR}}=1731 \pm 193 \mathrm{Ma}$ and $T_{\mathrm{DM}(\mathrm{DP})}=2021 \pm 215 \mathrm{Ma}$ ( $n=34$ values) (Clifford et al., 1995; Geringer et al., 1998).

Our new data show variations within the same intrusion. In Wheal Julia, $\varepsilon_{\mathrm{Nd}(1030 \mathrm{Ma})}$ varies from -7.2 to -10.7 and $\mathrm{Sr}_{\mathrm{i}}$ between 0.717 and 0.731 ; a similar variation in $\varepsilon_{\mathrm{Nd}(1030 \mathrm{Ma})}$ from -8.3 to -11.1 is observed within Jubilee with lower $\mathrm{Sr}_{\mathrm{i}}$ varying from 0.713 to 0.715. On the other hand Carolusberg is more homogeneous with $\varepsilon_{\mathrm{Nd}(1030 \mathrm{Ma})}$ from -7.3 to -8.6 , and $\mathrm{Sr}_{\mathrm{i}}$ in the range $0.716-0.719$.

\section{Discussion}

\subsection{Granites and syenite}

Our new geochemical data on the Concordia Granite as well as locally occurring garnet point to S-type affinities, as already concluded by Raith (1995). The high $\mathrm{K}_{2} \mathrm{O}$ content indicates a pelite-derived melt composition (Patiño-Douce and Johnston, 1991) with complete breakdown of muscovite and biotite in vapor-absent conditions, leaving a plagioclase- and $\mathrm{K}$ feldspar-bearing residue (Harris and Inger, 1992). The contents in $\mathrm{Rb}(>100 \mathrm{ppm}), \mathrm{Sr}(<300-400 \mathrm{ppm})$ and $\mathrm{Ba}$ $(<600-1000 \mathrm{ppm})$, with $\mathrm{Rb} /$ Ba ratio $>0.25$ also point to a pelitic source (Miller, 1985). An equilibrium batch melting model of a pelitic composition (Condie, 1993) using the parameters of Harris and Inger (1992) reproduces the range of values observed in the Concordia Granite.

The same mechanism of formation can be invoked for the Kweekfontein Granite, the typically high $\mathrm{La} / \mathrm{Yb}$ ratios being explained by fractionation of very small amounts of accessory minerals, such as xenotime, with high REE partition coefficients and much higher values for the HREE than for the LREE. As an alternative, a similar distribution can be obtained if garnet remains 


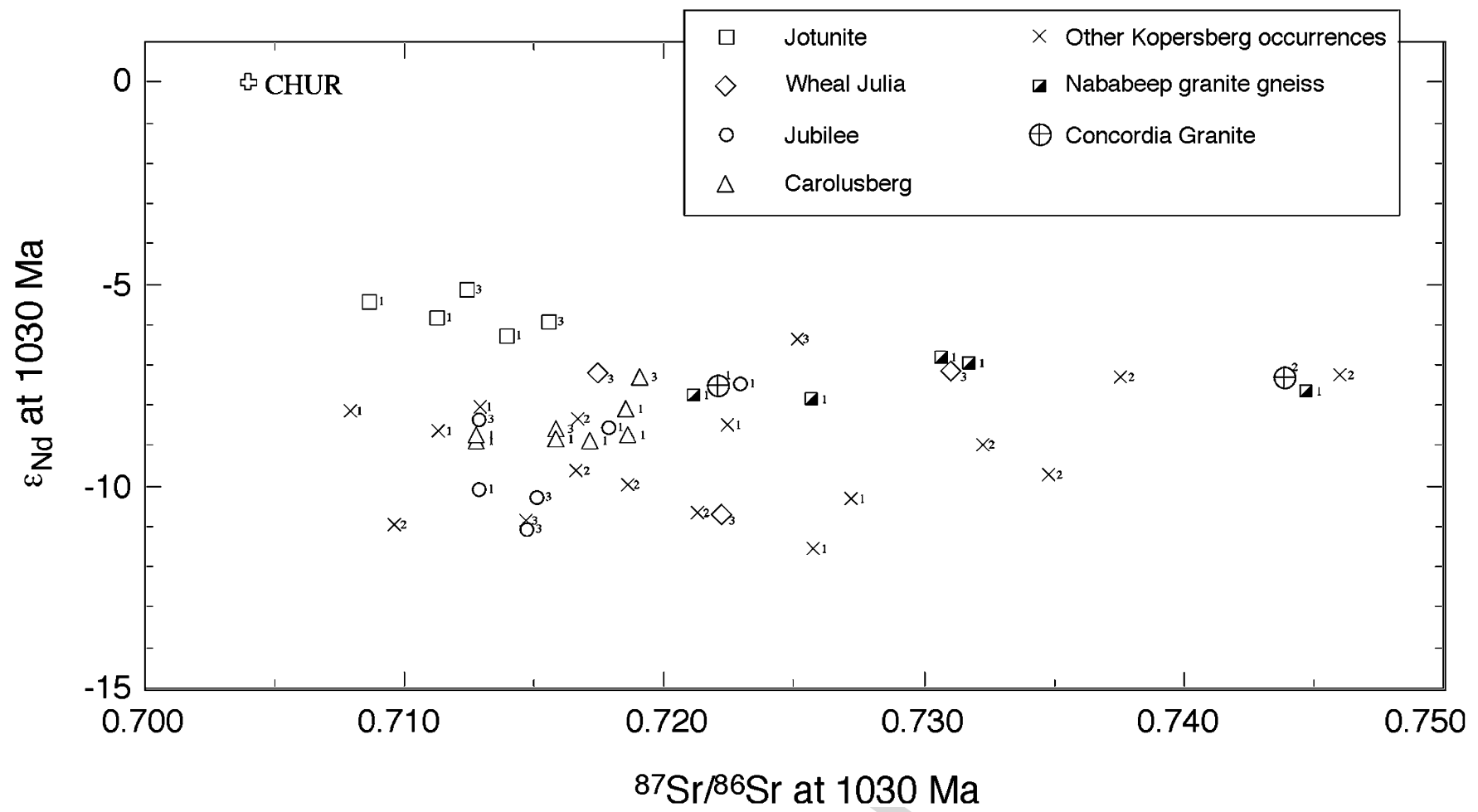

Fig. 9. $\varepsilon_{\mathrm{Nd}}$ vs. Sr isotope ratio at $1030 \mathrm{Ma}$ for samples from the O'okiep District. Source of data: 1, Clifford et al., 1995; 2, Geringer et al., 1998; 3 , new data from this work.

in the residue at the completion of the partial melting process.

Melting of K-rich andesites is a possible mechanism for the generation of the potassium-rich Rietberg granite (Roberts and Clemens, 1993). It should also be noted that the O'okiep jotunites and the Rietberg Granite show a Fe\# in the same range (AFM triangle in Fig. 3). Moreover, the REE distribution in the Rietberg Granite average composition (Fig. 4c) is subparallel to the Jubilee jotunitic melt obtained by inversion of the plagioclase composition (see below). It is therefore possible that the Rietberg Granite and the Koperberg Suite belong to the same magmatic series.

In the Rietberg Granite, the REE evolution (Fig. 4b) supports the following qualitative model of evolution that is inspired by current modelling of $\mathrm{K}_{2} \mathrm{O}$-rich granitic series (e.g. Duchesne et al., 1998; Vander Auwera et al., 2003). Commencing with a melt with composition of sample 20 (with $63.5 \% \mathrm{SiO}_{2}$ ) and subtracting an REE-poor cumulate increases the REE content to that of sample $22\left(67.2 \% \mathrm{SiO}_{2}\right)$. A REE-rich cumulate (somewhat similar to the syenite but with a positive $\mathrm{Eu}$ anomaly) may then crystallize to decrease the LREE to the final stage of evolution (sample 24 with $72.4 \% \mathrm{SiO}_{2}$ and a marked negative Eu anomaly of 0.26). More data are obviously needed to better constrain this model, but it is clear that the overall decrease of $\mathrm{P}_{2} \mathrm{O}_{5}$ with $\mathrm{SiO}_{2}$ in the
Rietberg Granite trend implies the presence of apatite at the liquidus and in the subtracted cumulate. This feature plays an important role in the discussion of a possible genetic link between the Rietberg and Concordia Granites. In Harker diagrams (Fig. 3), the Rietberg Granite trend appears to merge into the Concordia Granite compositions. Both granites are also very rich in $\mathrm{U}$ and $\mathrm{Th}$, but they display significant differences in the REE distribution (Fig. 4c). The negative Eu anomaly is more pronounced in Rietberg Granite, and the Concordia Granite is poorer in LREE but remarkably richer in HREE than the Rietberg Granite. The presence of apatite in the subtracted cumulate buffers the REE evolution in the Rietberg Granite magma, making a change in the $\mathrm{La} / \mathrm{Yb}$ ratio and the Eu anomaly very unlikely. This is in conflict with the views of McCarthy (1976) who suggested that a crystallization process links the two granites, but supports the age difference recognized by Clifford et al. (2004) on the basis of SHRIMP zircon ages.

The syenite (sample 65) may also be considered as belonging to the Rietberg Granite series because, for most elements, its composition plots on the extension of the Rietberg Granite range (Fig. 3). It can be considered with Rietberg Granite sample 107 since they both show high $\mathrm{K}_{2} \mathrm{O}$ contents, due to accumulation of $\mathrm{K}$-feldspar, a feature corroborated in the syenite by a very high $\mathrm{Ba}$ content (6064 ppm; Table 1). Interestingly, the positive 
$\mathrm{Eu}$ anomaly which should result from accumulation of feldspar is not observed in both cumulates (Fig. 4b) but, as shown by the high $\mathrm{P}_{2} \mathrm{O}_{5}$ contents in both rocks (up to $2 \%$ in syenite; Table 1 ), these cumulates also contain apatite, a REE-rich mineral with a marked negative Eu anomaly, which most certainly balances the positive anomaly in feldspars. This buffering role of apatite has been well documented in the jotunitic liquid line of descent (Wilmart et al., 1989; Duchesne, 1990; Vander Auwera et al., 1998b).

\subsection{Koperberg Suite}

\subsubsection{Jotunites}

Because of their igneous character and age, the jotunites can be considered as members of the Koperberg Suite. They are key to understanding the origin of the suite because similar rocks have been identified in Norway as parental melts to the AMCG suite (Duchesne et al., 1974, 1989; Demaiffe and Hertogen, 1981; Vander Auwera et al., 1998b). Here, the jotunite texture, mineral and chemical compositions, in particular the REE distributions (Fig. 6), are comparable to the Norwegian occurrences, and fall close to the range defined by primitive and evolved jotunites (sensu Vander Auwera et al., 1998b).

The jotunitic melt crystallization for sample 36, when modelled with Ghiorso algorithm (Ghiorso and Sack, 1995) at $5 \mathrm{kbar}$ and oxygen fugacities at the QFM buffer, shows clinopyroxene as the first mineral at the liquidus at $1200^{\circ} \mathrm{C}$, rapidly followed at $1180^{\circ} \mathrm{C}$ by plagioclase $\mathrm{An}_{53}$, and at $1150^{\circ} \mathrm{C}$ by apatite. Similar modelling for sample 53 shows that the melt at $1200^{\circ} \mathrm{C}$ was saturated in apatite and clinopyroxene, and plagioclase $\mathrm{An}_{57}$ appears at $1170^{\circ} \mathrm{C}$. The liquidus plagioclase compositions are slightly richer in An than the cumulus plagioclase compositional range $\left(\mathrm{An}_{30-50}\right)$ of the Koperberg Suite, but within error of the method. It is worth mentioning that at $5 \mathrm{kbar}$ the TJ jotunite from Rogaland, experimentally studied by Vander Auwera and Longhi (1994), is in equilibrium with an $\mathrm{An}_{48}$ plagioclase at $1050{ }^{\circ} \mathrm{C}$. It is thus concluded that jotunites belong to the same magma series as the Koperberg Suite and can be parental to the various lithologies.

\subsubsection{Cumulate rocks}

In Harker diagrams (Fig. 7), anorthosite and leuconorite plot close to the plagioclase pole, consistent with their petrographic definition and in agreement with their cumulate origin. Norites, on the other hand, are closer to the jotunitic liquid line of descent and therefore their interpretation as cumulates is ambiguous; they

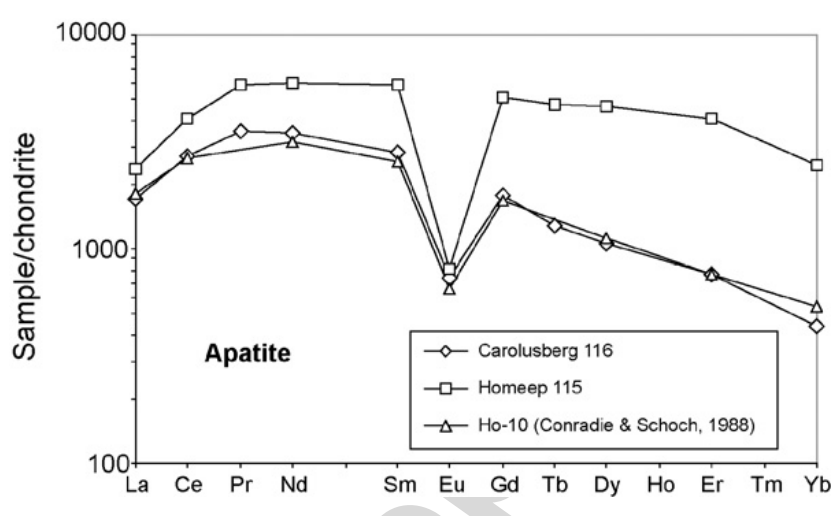

Fig. 10. Chondrite-normalized REE distribution in separated apatites from norite 116 from Carolusberg and magnetitite 115 from Homeep (see Table 4 for the analyses), compared with an analysis of an apatite from diorite Ho-10 by Conradie and Schoch (1988, p. 414).

may indeed contain a larger proportion of cumulate mafic minerals or of trapped intercumulus liquid. In contrast the jotunites plot on the liquid line of descent (Fig. 7) for nearly all elements (except $\mathrm{CaO}, \mathrm{MgO}, \mathrm{K}_{2} \mathrm{O}$ and $\mathrm{P}_{2} \mathrm{O}_{5}$ which are close to), and can therefore be considered as liquids in complete agreement with the chilled texture displayed by one of them (sample 53). The relatively low $\mathrm{P}_{2} \mathrm{O}_{5}$ and high $\mathrm{MgO}$ contents (Fig. 7) point to primitive jotunite, sensu Vander Auwera et al. (1998b). Finally, biotite diorite plot over a much larger area in the various diagrams (Fig. 7), making the identification of their cumulate or liquid character more difficult.

As expected in cumulate rocks, the REE distribution and content depend on the nature and proportion of cumulus minerals and intercumulus melt. In the present case, apatite can be surmised to have played a leading role, either as a cumulus mineral or as a mineral crystallized from the intercumulus trapped liquid. To test that hypothesis, we have separated and analysed apatite from two rocks (norite sample 116 from Carolusberg, and magnetitite sample 115 from Homeep East; Table 4) in which the amount of apatite is so high that it is very likely a cumulate (liquidus) mineral. The REE distribution (Fig. 10) in apatite shows very high contents and strong negative $\mathrm{Eu}$ anomalies, features that have already been reported by Conradie and Schoch (1988). In a diagram relating Ce versus $\mathrm{P}_{2} \mathrm{O}_{5}$ whole-rock contents (Fig. 11), apatite (sample 115) falls on the prolongation of a line passing through magnetitite, glimmerite, biotite diorite and some of the anorthosites, while apatite 116 (and apatite Ho-54 from Conradie and Schoch, 1988) plots on the regression line grossly passing through hypersthenite and melanorite. This suggests that the most important factors that control the REE contents of a sample are its apatite content and composition. 


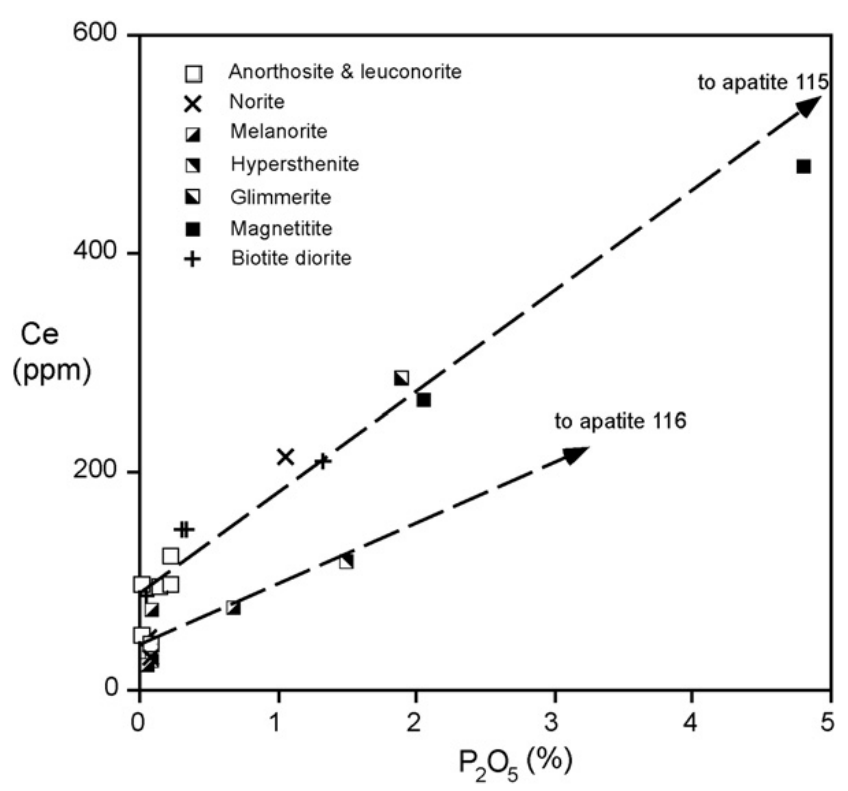

Fig. 11. Relationship between whole-rock $\mathrm{Ce}$ and $\mathrm{P}_{2} \mathrm{O}_{5}$ contents; the dashed lines point towards apatite compositions 115 and 116 (see Table 4). Note that the biotite diorite 112 with $\mathrm{Zr}$ value at $1173 \mathrm{ppm}$ falls outside the diagram.

Other trace element contents are also strongly controlled by the nature and proportion of the cumulus minerals. Hypersthenite, melanorite and glimmerite are rich in $\mathrm{Cu}, \mathrm{Ni}, \mathrm{Co}$ and $\mathrm{Cr}$, reflecting the high contents of $\mathrm{Cr}$ and $\mathrm{Ni}$ in hypersthene and biotite (Conradie and Schoch, 1986; Cawthorn and Meyer, 1993) and the association of $\mathrm{Cu}$-sulphide with hypersthene (Van Zwieten et al., 1996), a well-known characteristic to the mining geologists (Strauss, 1941). Magnetitite is particularly enriched in $\mathrm{V}$ and $\mathrm{Zn}$, consistent with the ability of these elements to enter magnetite.

\subsubsection{Inversion of plagioclase composition}

In cumulate rocks, the trace element content in a mineral is proportional to its content in the melt, but it also depends on the amount of melt that has been trapped in the cumulate (the trapped melt fraction of Irvine, 1982). Because mineral/melt distribution coefficients $(D)$ are reasonably well known, and provided the effects of the trapped melt fraction can be accounted for, it is thus theoretically possible to calculate the composition of the melt from the composition of any cumulus mineral.

Post-cumulus modification of the cumulus mineral composition has been addressed by numerous authors (cf. Irvine, 1980; Barnes, 1986; Bédard, 1994; Cawthorn, 1996). Highly incompatible elements are several orders of magnitude more enriched in the trapped melt than in the cumulus minerals. When the trapped melt fraction crystallizes, these elements are forced into the cumulus mineral overgrowths and, depending on their diffusivity, can migrate from margin to core to homogenize the grain contents. Bédard (1994) has, however, pointed out that when a trace mineral such as apatite or zircon crystallizes from the trapped melt fraction, the re-equilibration process is quite different. In the evolution of the trapped melt, the incompatible element content will increase up to a maximum value determined by the precipitation of the trace mineral which incorporates the element. In other words the incompatibility of the element changes to a compatible character and its content rapidly decreases in the further evolution of the trapped liquid. It is therefore very likely that the re-equilibration of cumulus mineral composition becomes negligible, in which case it is possible to calculate the co-existing melt composition by inversion of cumulus mineral composition.

We have adopted this approach here because, as already mentioned, apatite is an ubiquitous trace mineral in the Koperberg anorthosite, leuconorite, and norite (except in norite 115 where apatite is so abundant that it is likely a liquidus/cumulus mineral). We have chosen plagioclase as the cumulus mineral for study, but a difficulty of the method is the possible contamination of the separated fraction by composite grains with apatite. To solve this problem, we have used a selective dissolution technique to remove the traces of apatite in plagioclase concentrates (see Appendix 2).

The trace element content of the separated plagioclase from cumulate rocks is given in Table 3 and illustrated in Fig. 12. The anorthite content in plagioclase varies between $\mathrm{An}_{36}$ and $\mathrm{An}_{48}$. There is a crude negative correlation of the An content with $\mathrm{K}, \mathrm{Rb}$, and $\mathrm{Ba}$, and $\mathrm{Sr}$ is not correlated with $\mathrm{Ca}$ and varies in the same occurrence by nearly a factor of 2 , a feature common in anorthosite plagioclase (Duchesne, 1978; Vander Auwera et al., 2000). These overall variations very likely reflect variations in the degree of differentiation of the samples. The REE distributions in plagioclase show positive Eu anomalies, with $\mathrm{Eu} / \mathrm{Eu}^{*}$ ratios varying between 2 and 5 and roughly decreasing with the An content. The $[\mathrm{La} / \mathrm{Yb}]_{n}$ ratios are high and variable (89-349) and grossly correlate positively with the $\mathrm{Eu} / \mathrm{Eu}^{*}$ ratio (Fig. 12). It is striking that samples coming from the same occurrence (samples 66 and 116 from Carolusberg, 108 and 110 from Wheal Julia, 111 and 113 from Jubilee) show rather similar REE distributions, particularly the $\mathrm{Eu} / \mathrm{Eu}^{*}$ and $[\mathrm{La} / \mathrm{Yb}]_{n}$ ratios, whatever the petrographic type. This indicates that the REE evolution was probably buffered during the differentiation process characteristic of each intrusion. In a fractional crystallization process, this would imply a minerals/melt bulk partition coefficient of the REE close to unity, which in turn would suggest the presence of apatite in the cumulus mineral assemblage. 

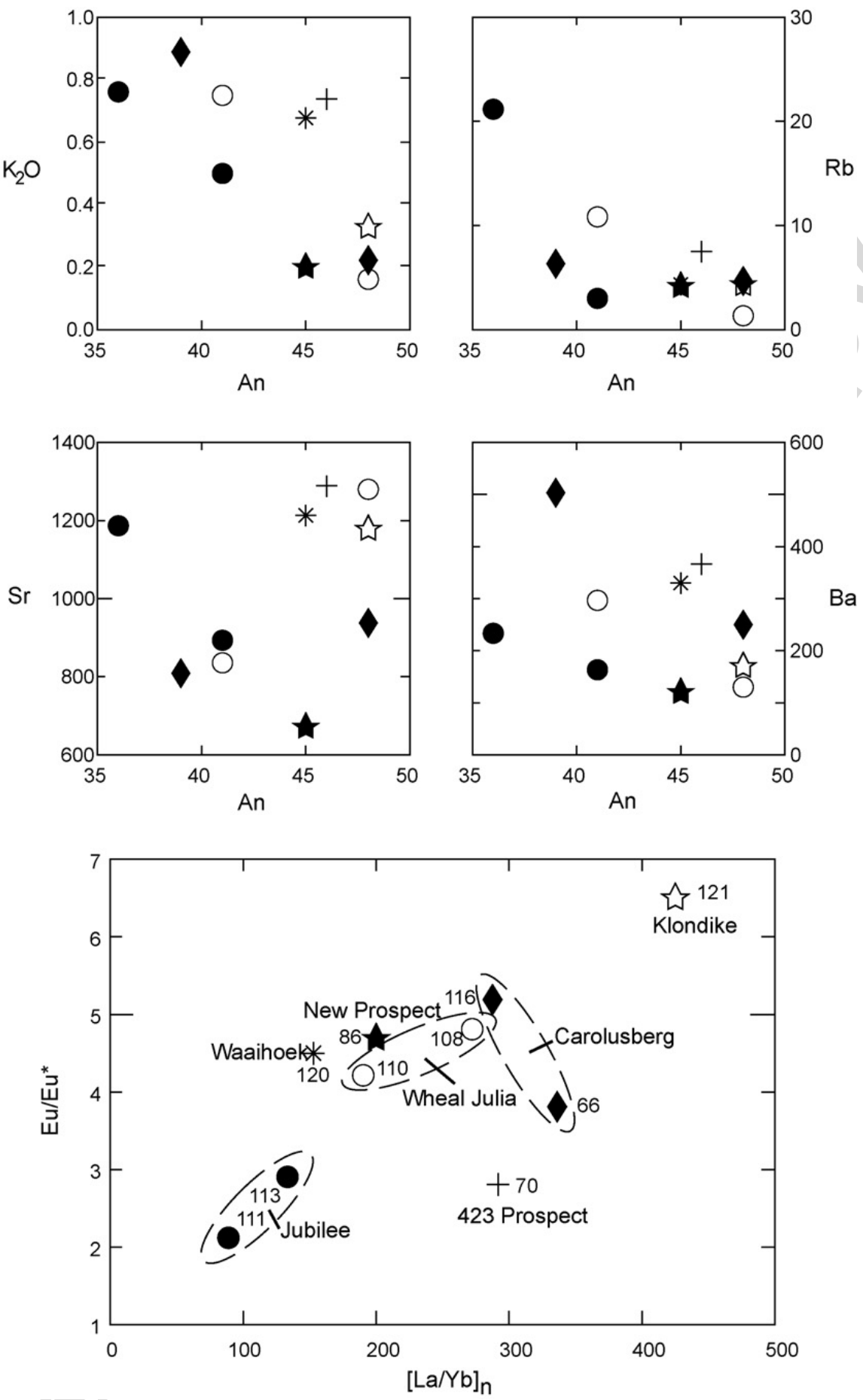

Fig. 12. Trace element composition of plagioclase separated from cumulate rocks from various occurrences of the Koperberg Suite in the O'okiep District (analyses in Table 3).

The compositions of the cumulus plagioclase may now be used to calculate the REE concentrations in the melts in equilibrium with the cumulates. To successfully invert the plagioclase composition, an appropriate set of plagioclase/melt partition coefficients for the REE $(D)$ is required. The interval of variation of $D$ for plagioclase co-existing with andesitic (intermediate) liquids reported in the literature (see Henderson, 1982; Rollinson, 1993) is more restricted in composition than for basaltic liquids. Thus an arbitrary choice of a set of $D$ within that range will not seriously bias the results. The values adopted (see Table 7) are those used in the modelling of the jotunite evolution (Vander Auwera et al., 1998b); they are close to values generally accepted in modelling (see e.g. Martin, 1987). Results of the inversion are given in Table 7 and Fig. 13. 


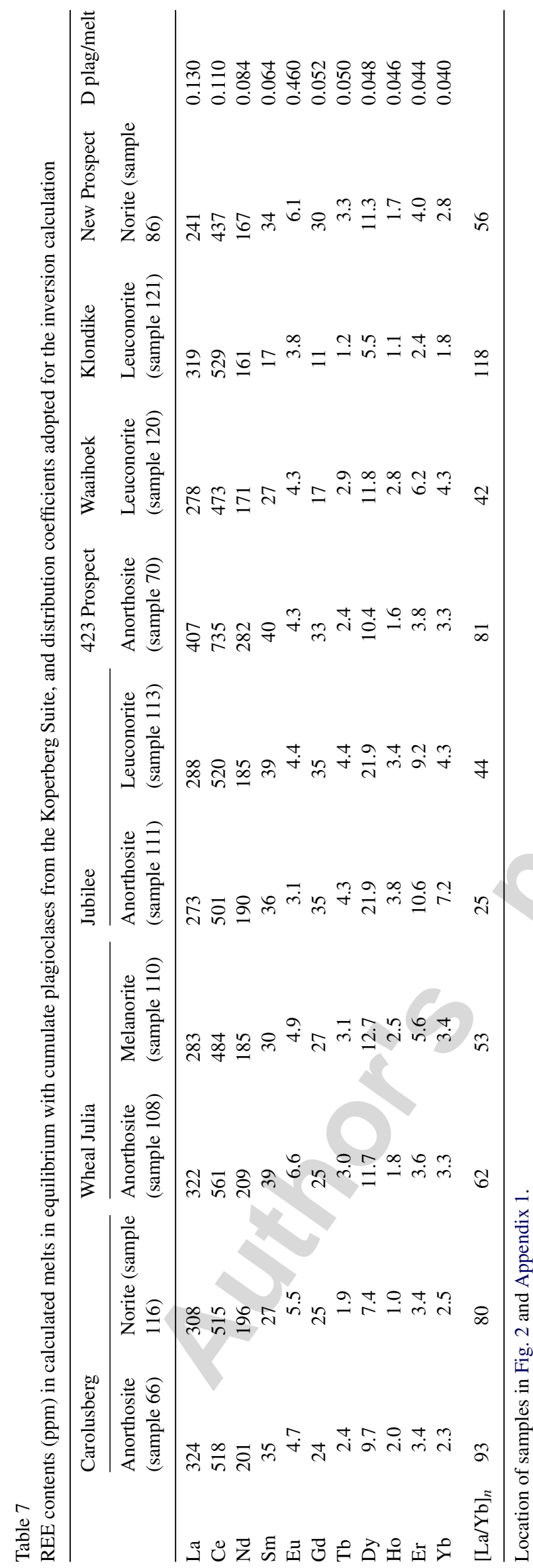

The LREE abundances in the recalculated melts (Fig. 13) show similar patterns and small variation in content (by a factor 1.7), while the HREE are more dispersed and vary by a factor of 5. The well-documented intrusions (Carolusberg, Wheal Julia and Jubilee) typically show identical LREE and variable HREE, with $[\mathrm{La} / \mathrm{Yb}]_{n}$ varying between $\approx 100$ and $\approx 280$. These features constrain the melting process and the mineralogy of the source which melts. For low degree of melt fraction $F$, the equation of equilibrium partial melting becomes $c_{\mathrm{L}}=c_{0} / D_{0}$, in which $c_{\mathrm{L}}$ and $c_{0}$ are the element content in the melt and the source, respectively, and $D_{0}$ the mineral/melt bulk partition coefficient. Assuming a common source of the three magma batches (i.e. $c_{0}$ constant), $c_{\mathrm{L}}$ will essentially vary with $D_{0}$. Small variation in the amount of garnet in the source (e.g. from 5\% to $10 \%$ ) has a drastic influence on the HREE bulk partition coefficient without changing the LREE partition coefficients, because garnet has REE partition coefficients which rapidly increase from $\mathrm{La}$ to $\mathrm{Lu}$. Small degree of melting and small, but variable, amounts of garnet in the source are thus suggested in order to produce jotunitic melts with variable $\mathrm{La} / \mathrm{Yb}$ ratios.

REE distributions in jotunitic melts calculated by inversion (Table 7) are compared to those observed in jotunite samples $(36,53)$ in Fig. 13b. The difference between the two types of distribution is striking for the LREE, which are clearly less abundant in the jotunite samples. This can mainly result from differences in the source rock composition and in the degree of melting, factors which are not much constrained. A simple calculation of an equilibrium partial melting model shows that using partition coefficients of Martin (1987), with a source rock made up of $40 \%$ plagioclase $+35 \%$ clinopyroxene $+20 \%$ orthopyroxene $+5 \%$ garnet, a variation in the degree of melting $\mathrm{F}$ from $1 \%$ to $40 \%$ can reproduce the calculated and observed REE distributions, respectively. Since at or near eutectic compositions the melt does not change its major element composition, such a model, though loosely constrained, appears plausible.

\subsubsection{Biotite diorite composition}

As already mentioned, the position of biotite diorites in Harker diagrams (Fig. 7) is ambiguous and it is not possible to decide whether they are cumulates, liquids or crystal-laden liquids. Except for sample 112 (Jubilee), trace elements in plagioclase (Table 3) are not essentially different from the other cumulus plagioclases; REE contents, Eu positive anomalies and $\mathrm{La} / \mathrm{Yb}$ ratios fall within or close to the same ranges. The somewhat more albitic character of some plagioclases tends to indicate slightly more evolved characters than the other rocks. 


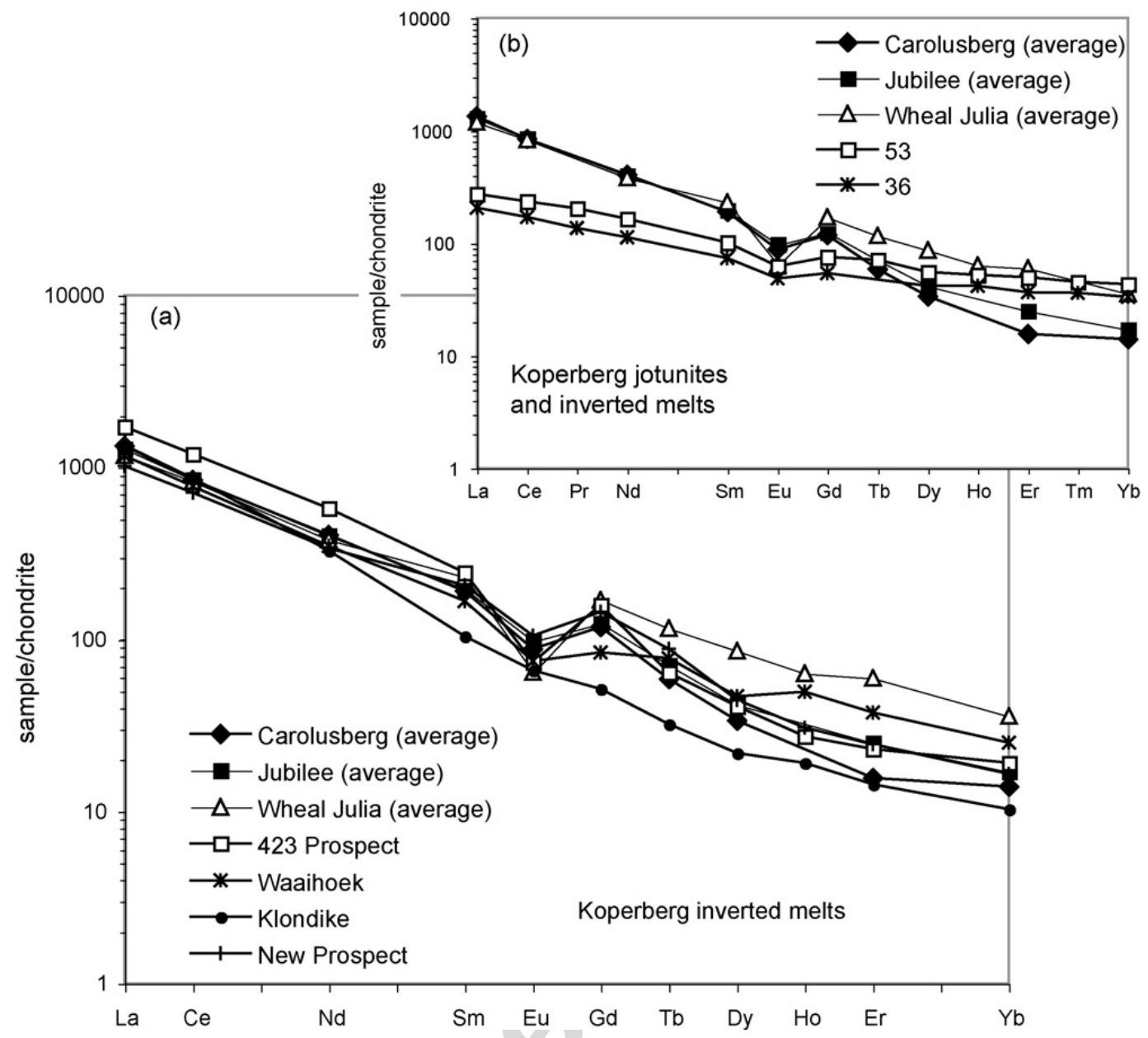

Fig. 13. Calculated REE distribution in melts in equilibrium with cumulus plagioclases (data in Table 7): (a) from seven occurrences-the REE contents from the same intrusion (Carolusberg, Jubilee, Wheal Julia), very similar, have been averaged; (b) Koperberg jotunite (samples 36 and 53 , Table 5) compared to the Carolusberg, Jubilee, and Wheal Julia average distributions.

Whole-rock biotite diorite composition from sample 112 (Table 2) is also different from the other biotite diorite by several features. It shows a large $\mathrm{Zr}$ content (1173 ppm), possibly reflecting zircon crystallization at the liquidus and its accumulation, thus explaining zircon inclusions in the plagioclase and high $\mathrm{Zr}$ content in the separated plagioclase (Table 3). Th content is extremely high $(418 \mathrm{ppm})$ (partly due to zircon accumulation?). The plagioclase REE distribution (Table 3) is higher by a factor of 3 than in sample 78 , another specimen from the same intrusion. The whole-rock REE abundances of sample 112 (Fig. 8) are also higher (up to 6-10 times those of the other biotite diorites) with the largest negative Eu anomaly. Some other biotite diorites have also peculiar trace element contents (Table 2): sample 109 (Wheal Julia) is also Th rich (53 ppm) and displays the maximum Cs value (4.7 ppm) in the Kopperberg Suite; sample 118 (Dixon's Farm) is anomalously Ba rich (4844 ppm). Biotite diorite thus constitute a geochemically heterogeneous group probably deriving from melts with different trace element contents, and variously laden with cumulus minerals.

\subsubsection{Isotopic composition (Fig. 9)}

Clifford et al. (1995) have emphasized that the overall isotopic characteristics of the Koperberg Suite and their country rocks support a major crust-forming event at the end of Paleoproterozoic times, and that the Koperberg Suite was derived from a crustal progenitor in the lower crust. Geringer et al. (1998), whilst not rejecting this model, preferred the alternative explanation of a mantle-derived Koperberg magma contaminated by a significant amount of crustal material. To account for the negative $\varepsilon_{\mathrm{Nd}}$ values the mantle would have been either highly enriched or the melting product would have been contaminated by Archaean felsic crust with lower negative $\varepsilon_{\mathrm{Nd}}$ values. In both cases, the high $\mathrm{Sr}$ initial ratios would result from an additional assimilation with Proterozoic felsic crust. A highly enriched mantle source, equivalent to EMI I (Rollinson, 1993), is plausible, but 
the weakness of the model lies in the uncertainty about the nature of the felsic contaminant: (1) an Archaean crust has never been recorded by age determination in Namaqualand (Clifford et al., 1995, 2004), the SHRIMP ages of cores from country rocks zircons are never older than 2050 Ma (Clifford et al., 2004); (2) the Proterozoic contaminant invoked by Geringer et al. (1998) with $\varepsilon_{\mathrm{Nd}}=-2$ and $\mathrm{Sr}_{\mathrm{i}} c a .0 .760$ is unrealistic for a Proterozoic material (cf. Rollinson, 1993).

Attempts to explain the $\mathrm{Sr}_{\mathrm{i}}$ variations within a single ore-body of the Koperberg Suite were proposed by Brandriss and Cawthorn (1996) and Van Zwieten et al. (1996) for the Hoogkraal and Jubilee intrusions, respectively. We agree with this interpretation that contamination of a mafic, less radiogenic magma by crustal material took place either by local interaction with country rocks in the magma chamber or in the structural conduit exploited by the crystal mush en route to the final level of emplacement. Moreover we concur with these authors that the felsic contaminant was not to be found in the country rocks, because the latter were not radiogenic enough, and particularly inadequate to account for $\mathrm{Sr}_{\mathrm{i}}$ values up to 0.748 (see Fig. 9). We conclude, therefore, that partial melting of the underlying 2000-Ma-old lower crust is the process most likely to have generated the Klondikean magmatism (cf. Clifford et al., 2004).

To explain the negative $\varepsilon_{\mathrm{Nd}}$ and the large variation of $\mathrm{Sr}_{i}$ between the various Koperberg intrusions, we propose the following scenario which is inspired from similar cases in the Rogaland (Schiellerup et al., 2000) and Polish (Wiszniewska et al., 2002) anorthosites: a magma was extracted from the mantle at an age $c a$. $1900 \mathrm{Ma}$, corresponding to the mean age of zircon cores in O'okiep gneiss and granite (Clifford et al., 2004), and intermediate between the mean $T_{\mathrm{CHUR}}$ and $T_{\mathrm{DM}} \mathrm{Nd}-$ model ages of all analysed O'okiep rocks. This magma crystallized to build up an oceanic crust with a REE distribution enriched in LREE (thus with low $\mathrm{Sm} / \mathrm{Nd}$ ratios), that was subsequently downthrust and metamorphosed in granulite facies to become part of the deep crust (see below for the actual process). The resulting plagioclase + pyroxenes mafic granulite inherited the $\mathrm{Sr}_{\mathrm{i}}$ heterogeneities of its progenitor. These heterogeneities can be accounted for if the oceanic crust $\left(\varepsilon_{\mathrm{Nd}}=+3.5\right.$ and $\mathrm{Sr}_{\mathrm{i}}=0.7018$ at $1900 \mathrm{Ma}$ ) had variable $\mathrm{Sm} / \mathrm{Nd}$ ratios and was altered by hydrothermal circulation to produce variable $\mathrm{Rb} / \mathrm{Sr}$ ratios. A simple calculation (Table 8 ) shows indeed that a range of $\mathrm{Rb} / \mathrm{Sr}$ values from 0.1 to 1.2 can produced, after a $900 \mathrm{Myr}$ evolution, the range of $\mathrm{Sr}_{\mathrm{i}}$ from 0.709 to 0.748 observed in the Koperberg Suite (Fig. 14). Such a range of $\mathrm{Rb} / \mathrm{Sr}$ ratios is observed e.g. in DSDP leg 417 of the Deep Sea Drilling Project (Alt

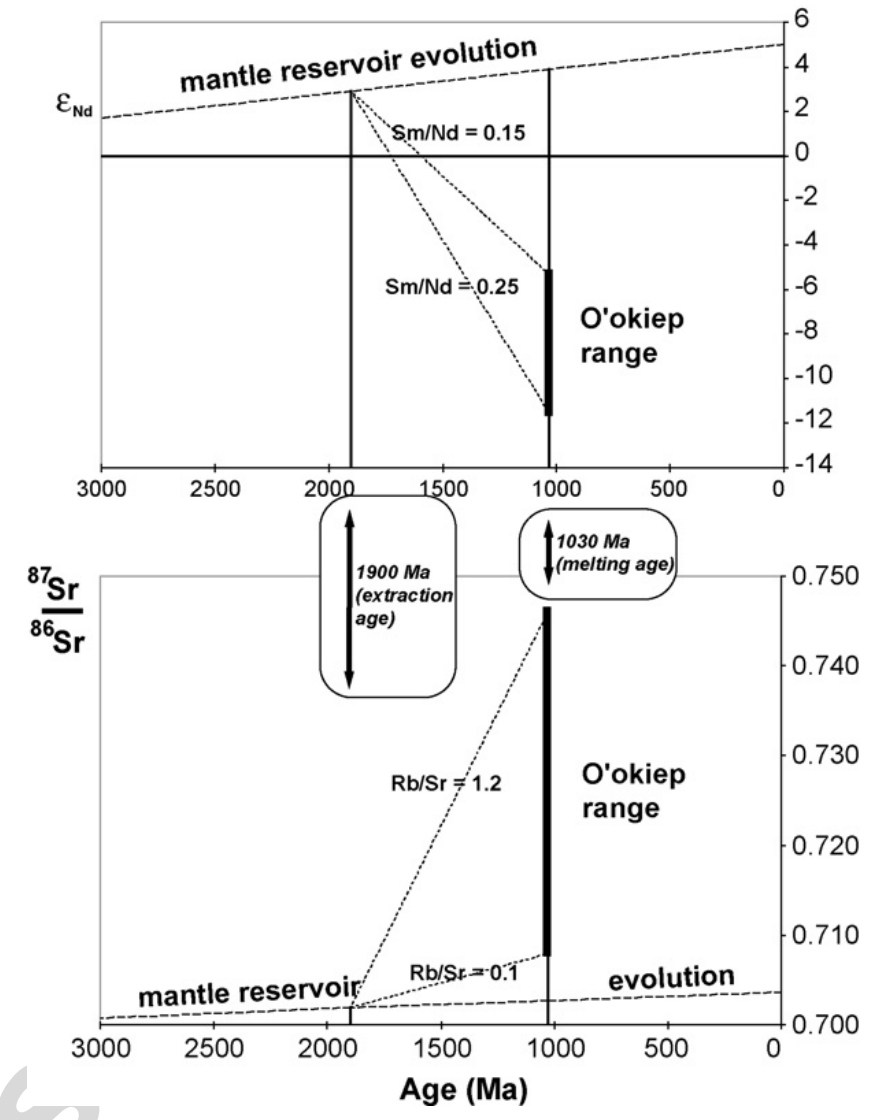

Fig. 14. Initial $\varepsilon_{\mathrm{Nd}}$ and ${ }^{87} \mathrm{Sr} /{ }^{86} \mathrm{Sr}$ isotope ratio vs. age evolution for the O'okiep District (see text).

and Honnorez, 1984). The hydrothermal alteration of the progenitor may have been accompanied by the formation of massive $\mathrm{Fe}-\mathrm{Cu}$-sulphide deposits, such as the TAG hydrothermal mound (Rona et al., 1986), possibly remobilized during the melting process to form the Koperberg deposits. In such a scenario, to account for the negative $\varepsilon_{\mathrm{Nd}}$ values $(-8.6 \pm 1.6)$ of the Koperberg Suite (Fig. 14), the oceanic crust must have a LREE enriched distribution. Our calculation (Table 8) shows that the progenitor must have had a range of $\mathrm{Sm} / \mathrm{Nd}$ ratios between 0.16 and 0.25 . Such ratios can be found in enriched MORB (E-MORB) or in the Azores oceanic islands where $\mathrm{Sm} / \mathrm{Nd}$ of $0.21 \pm 0.04$ are reported (White et al., 1979). It can thus be concluded that alteration of an E-MORB oceanic crust can produce a possible progenitor for the Kopersberg Suite and associated $\mathrm{Cu}$ deposits. This inference has been also proposed among other possible scenarios, but without isotopic constraints, for the Caraiba $\mathrm{Cu}$-rich hypersthenite-norite complex (Oliveira and Tarney, 1995).

\subsection{Origin of the O'okiep plutonism}

We propose that the formation of the 1900-Ma-old oceanic crust was related to the Eburnian event, dur- 
Table 8

Recalculated values of $\mathrm{Rb} / \mathrm{Sr}$ and $\mathrm{Sm} / \mathrm{Nd}$ ratios in the inferred gabbroic progenitor at $1900 \mathrm{Ma}$, the extraction age from the mantle, to account for the isotopic ratios at $1030 \mathrm{Ma}$

\begin{tabular}{|c|c|c|c|c|c|}
\hline & \multicolumn{2}{|c|}{$\left({ }^{87} \mathrm{Sr} /{ }^{86} \mathrm{Sr}\right)$ at 1030} & $\left({ }^{87} \mathrm{Sr} /{ }^{86} \mathrm{Sr}\right)_{\mathrm{CHUR}}$ at 1900 & ${ }^{87} \mathrm{Rb} /{ }^{86} \mathrm{Sr}$ & $\mathrm{Rb} / \mathrm{Sr}$ \\
\hline Average & \multicolumn{2}{|c|}{0.720438} & 0.701965 & 1.4861 & 0.5128 \\
\hline Maximum & \multicolumn{2}{|c|}{0.746012} & 0.701965 & 3.5435 & 1.2201 \\
\hline \multirow[t]{2}{*}{ Minimum } & \multicolumn{2}{|c|}{0.707911} & 0.701965 & 0.4784 & 0.1653 \\
\hline & $\varepsilon_{\mathrm{Nd}}$ at 1030 & $\left({ }^{143} \mathrm{Nd} /{ }^{144} \mathrm{Nd}\right)_{\text {at } 1030}$ & $\left({ }^{143} \mathrm{Nd} /{ }^{144} \mathrm{Nd}\right)_{\mathrm{CHUR}}$ at 1900 & ${ }^{147} \mathrm{Sm} /{ }^{144} \mathrm{Nd}$ & $\mathrm{Sm} / \mathrm{Nd}$ \\
\hline Average & -8.6 & 0.510869 & 0.510179 & 0.1210 & 0.20 \\
\hline Maximum & -5.33 & 0.511036 & 0.510179 & 0.1502 & 0.25 \\
\hline Minimum & -11.54 & 0.510718 & 0.510179 & 0.0945 & 0.16 \\
\hline
\end{tabular}

ing which the Richterveld terrane to the North was generated. Parts of that oceanic crust were incorporated to the lower crust by underthrusting, and then metamorphosed under granulite facies conditions during collision to become a mafic granulite. After a long period of quiescence lasting $c a$. $600 \mathrm{Myr}$, the Namaquan collision ( $c a .1300 \mathrm{Ma}$ ) took place to the NE of the region, a first melting event followed (O'okiepian Episode, ca. $1190 \mathrm{Ma}$ ) in a post-collisional setting, and a huge amount of granitic material was generated in the O'okiep District. Some $160 \mathrm{Ma}$ later a second melting event (Klondikean Episode, ca. $1030 \mathrm{Ma}$ ) affected the region, and the lower crustal mafic granulite was melted under dry conditions at $10-13$ kbar pressure to produce the Koperberg parental magma (Longhi et al., 1999).

This model implies episodic and strong increases of temperature to melt large volumes of material during the O'okiepian Episode and of mafic material during the Klondikean Episode. Additionally, an increase in pressure is needed to produce jotunite melts in the Klondikean Episode. A thermal plume (Clifford et al., 1995) would explain the temperature rises during the Klondikean Episode. Alternatively during both heating events, the asthenosphere might have been placed close to the base of the crust by linear delamination of the subcontinental lithosphere along a mega-shear zone $(c f$. Lachenbruch et al., 1985; Teyssier and Tikoff, 1998). Reactivation of a pre-existing shear zone could account for the episodic repetition of thermal events (Liégeois et al., 2003). As for the pressure, a thickened crust by intracontinental (Ampferer-type) subduction (Clifford et al., 2004) or a "crustal tongue" (Duchesne et al., 1999), resulting from the Eburnian subduction, can explain the necessary high values. Finally, the Klondikean melting process produced a number of individual magma batches that inherited the isotopic heterogeneities of the source rocks.

\section{Conclusions}

1. The Concordia and Kweekfontein Granites are high$\mathrm{K}$ granitic rocks produced by dehydration melting of metapelites. The Rietberg Granite is geochemically similar to typical post-collisional shoshonitic trends, such as the Rogaland charnockites and the Tismana granitic rocks. Its REE distribution suggests consanguinity with some of the Koperberg Suite melts. It belongs to the K-rich granitic kindred, which is best explained through partial melting of an andesitic crust in post-collisional settings (Roberts and Clemens, 1993; Liégeois et al., 1998).

2. Jotunites are members of the Koperberg Suite; they are identified geochemically as primitive jotunitic melts, similar to those found as chilled margin to several intrusions in the Rogaland anorthosite province. This constitutes the best unambiguous evidence that the Koperberg rock series belongs to the massif-type anorthosite kindred and to the AMCG suite. Following the work of Longhi et al. (1999) and Longhi (2005), jotunites derive from the melting in dry conditions (granulite facies) at high pressure (11-13 kbar; depth $40-50 \mathrm{~km}$ ) of a 2-pyroxene plagioclase rock, i.e. a mafic crustal rock. The occurrence of jotunite in the Koperberg Suite strongly corroborates the crustal rock origin, already deduced by Clifford et al. (1995) from the $\mathrm{Sr}, \mathrm{Nd}$ and $\mathrm{Pb}$ isotope systematics.

3. The Koperberg anorthosites and related rocks (including hypersthenite, glimmerite, and magnetitite) show a geochemistry compatible with an origin as cumulate rocks. Irrespective of the petrographic type, the REE geochemistry of the Koperberg cumulates is mostly controlled by their apatite content. One syenite also shows characteristics of a cumulate rocks derived from the Rietberg Granite magma. The origin of the biotite diorite whether as melts, cumulates or crystal-laden liquids remains ambiguous. 
4. In contrast to most massif-type anorthosites that show evidence of polybaric crystallization, the Koperberg anorthosites and related rocks were produced through crystallization in magma chambers or in conduits, possibly at medium pressure (3-5 kbar), followed by forceful injection of the cumulate crystal mush along steep structures.

5. Inversion modelling of plagioclase compositions permits the reconstruction of the melt compositions from which the anorthosites were derived. The REE distributions obtained for the melts characteristic of the different occurrences are similar for the LREE but show variable HREE contents (Fig. 13), suggesting small degrees of melting with small, but variable, amounts of garnet in the residual source rock. The melt compositions obtained by inversion of plagioclase compositions have REE distributions which significantly differ from those observed in primitive jotunite (Fig. 13), but this difference can be accounted for by different degrees of partial melting of the source rock.

6. The various intrusions of the Koperberg Suite show distinct and variable $\mathrm{Sr}-\mathrm{Nd}$ isotopic signatures. The negative $\varepsilon_{\mathrm{Nd}}(1030 \mathrm{Ma})$ can be simply explained by remelting an oceanic crust protolith with a EMORB REE distribution, such as the Azores Islands, extracted from the mantle at $1900 \mathrm{Ma}$. The large $\mathrm{Sr}_{\mathrm{i}}$ variation at $1030 \mathrm{Ma}$ could be the result of the hydrothermal alteration of the protolith at $1900 \mathrm{Ma}$, generating a range of $\mathrm{Rb} / \mathrm{Sr}$ ratios, which could be also at the origin of the $\mathrm{Cu}$-sulphide mineralization.

7. Final remark: The model we suggest here has the merit of invoking a simple but plausible mechanism (the melting of a crustal mafic source) to account for the very peculiar isotope composition of the Koperberg Suite that is supported by experimental work on the genesis of the anorthosite parent magma. Moreover the overall geodynamical framework implied by the model is also in good agreement with a recently developed concept on the evolution of cratons. Finally the intriguing occurrence of $\mathrm{Cu}$ sulphides associated with the Koperberg Suite is also explained.

\section{Acknowledgements}

The authors are grateful to the Jim and Gladys Taylor Educational Trust and to the Belgian Fund for Joint Research for funding of the ICP-MS and XRF analyses, and to Gold Fields of South Africa for logistics support. The geochemical analyses were carried on by G. Bologne who is greatly thanked. Three anonymous reviewers are thanked for their constructive suggestions.
Appendix A. O'okiep samples and localities (see Fig. 2)

\begin{tabular}{|c|c|c|c|}
\hline $\begin{array}{l}\text { Sample } \\
\text { number }\end{array}$ & $\begin{array}{l}\text { Locality } \\
\text { number }\end{array}$ & $\begin{array}{l}\text { Rock } \\
\text { type }\end{array}$ & Locality \\
\hline \multicolumn{4}{|c|}{ Granites and syenite } \\
\hline 20 & 23440 & Rietberg Granite & Jan Coetzee \\
\hline 22 & 23441 & Rietberg Granite & Steyerkraal \\
\hline 24 & 23443 & Rietberg Granite & Rietberg \\
\hline 25 & 23439 & Concordia Granite & Wheal Julia \\
\hline 60 & X20472 & Concordia Granite & O'okiep District \\
\hline 65 & $82 / \mathrm{A} / 21$ & Syenite & $\begin{array}{l}450-\mathrm{ft} \text { level, } \\
\text { Spektakel }\end{array}$ \\
\hline 105 & 96/A/4 & Concordia Granite & $\begin{array}{l}5 \mathrm{~km} \text { NNW of } \\
\text { Nababeep }\end{array}$ \\
\hline 106 & $97 / 1$ & $\begin{array}{l}\text { Kweekfontein } \\
\text { Granite }\end{array}$ & Kweekfontein \\
\hline 107 & 96/A/5 & Rietberg Granite & Jan Coetzee \\
\hline \multicolumn{4}{|c|}{ Koperberg Suite } \\
\hline 30 & $69 / \mathrm{A} / 26$ & Tonalite & Homeep East \\
\hline 36 & $73 / \mathrm{B} / 4$ & Jotunite & Narrowhuis \\
\hline 53 & $84 / \mathrm{A} / 26$ & Jotunite & Ratelkraal \\
\hline 66 & $84 / \mathrm{A} / 16 \mathrm{C}$ & $\begin{array}{l}\text { Andesine } \\
\text { anorthosite }\end{array}$ & $\begin{array}{l}\text { 2435-ft level, } \\
\text { Carolusberg West }\end{array}$ \\
\hline 70 & $85 / \mathrm{A} / 10$ & $\begin{array}{l}\text { Andesine } \\
\text { anorthosite }\end{array}$ & 423 Prospect \\
\hline 78 & $82 / \mathrm{A} / 6$ & Biotite diorite & Jubilee \\
\hline 79 & 85/A/6 & Biotite diorite & Jubilee \\
\hline 82 & 85/A/17 & $\begin{array}{l}\text { Magnetite- } \\
\text { hypersthenite }\end{array}$ & $\begin{array}{l}\text { Jan Coetzee South } \\
\text { West }\end{array}$ \\
\hline 85 & $82 / \mathrm{A} / 7$ & Norite & Jubilee \\
\hline 86 & $84 / \mathrm{A} / 5$ & Norite & New Prospect \\
\hline 87 & $85 / \mathrm{A} / 28$ & Melanorite & $\begin{array}{l}\text { 1214-ft level, } \\
\text { Carolusberg West }\end{array}$ \\
\hline 88 & $85 / \mathrm{A} / 25$ & Melanorite & $\begin{array}{l}\text { 945-ft level, } \\
\text { Carolusberg West }\end{array}$ \\
\hline 90 & $81 / \mathrm{A} / 9 \mathrm{a}$ & Melanorite & $\begin{array}{l}\text { 2435-ft level, } \\
\text { Carolusberg West }\end{array}$ \\
\hline 108 & $91 / \mathrm{A} / 2$ & $\begin{array}{l}\text { Andesine } \\
\text { anorthosite }\end{array}$ & Wheal Julia \\
\hline 109 & $91 / \mathrm{A} / 3$ & Biotite diorite & Wheal Julia \\
\hline 110 & $91 / \mathrm{A} / 5$ & Melanorite & Wheal Julia \\
\hline 111 & JCD96.4 & $\begin{array}{l}\text { Andesine } \\
\text { anorthosite }\end{array}$ & Jubilee \\
\hline 112 & JCD96.5 & Biotite diorite & Jubilee \\
\hline 113 & JCD96.7 & Leuconorite & Jubilee \\
\hline 114 & $91 / \mathrm{A} / 7$ & Biotite diorite & Homeep East \\
\hline 115 & JCD96.10 & Magnetitite & Homeep Easr \\
\hline 116 & 91/A/39 & Norite & $\begin{array}{l}1276 \mathrm{~m} \text { level, } \\
\text { Carolusberg West }\end{array}$ \\
\hline 117 & $91 / \mathrm{A} / 35$ & Hypersthenite & $\begin{array}{l}1510 \mathrm{~m} \text { level, } \\
\text { Carolusberg West }\end{array}$ \\
\hline 118 & 85/A/18(ii) & Biotite meladiorite & Dixon's Farm \\
\hline 119 & $73 / \mathrm{B} / 20$ & Leuconorite & Kliphoog North \\
\hline 120 & 73/B28 & Leuconorite & Waaihoek \\
\hline 121 & $91 / \mathrm{A} / 18$ & Leuconorite & Klondike \\
\hline 122 & 91/A/50 & Norite & $\begin{array}{l}\text { KC112, depth } \\
75 \mathrm{ft}, \text { Koperberg } \\
\text { Central }\end{array}$ \\
\hline
\end{tabular}


Appendix A (Continued)

\begin{tabular}{|c|c|c|c|}
\hline $\begin{array}{l}\text { Sample } \\
\text { number }\end{array}$ & $\begin{array}{l}\text { Locality } \\
\text { number }\end{array}$ & $\begin{array}{l}\text { Rock } \\
\text { type }\end{array}$ & Locality \\
\hline 123 & 91/A/21 & Glimmerite & Flat Mine North \\
\hline 125 & $84 / \mathrm{A} / 24$ & $\begin{array}{l}\text { Magnetite } \\
\text { hypersthenite }\end{array}$ & Ratelkraal \\
\hline 126 & JCD96.15 & Biotite diorite & Sugar loaf \\
\hline
\end{tabular}

\section{Appendix B. Methodology}

Plagioclase and apatite were separated and purified using standard techniques of mineral separation (dense liquids and Frantz Isodynamic separator). Plagioclase grains were etched in $\mathrm{HCl} 6 \mathrm{~N}$ solution in order to dissolve trace amounts of apatite as composite grains.

XRF was used to measure whole-rock major element compositions on Li-borate glass with a CGR ALPHA 2020 spectrometer following the method of Bologne and Duchesne (1991). ICP-MS (VG Plasma Quad PQ2) was used to analyse the trace elements including REE in plagioclase following the method of Vander Auwera et al. (1998a) on dissolved Li-borate beads.

$\mathrm{Sr}$ and $\mathrm{Nd}$ isotopic compositions of plagioclase were measured on a VG354 mass spectrometer at Johannesburg and the isotopic measurements on the two rock samples were made at the Université Libre de Bruxelles on a Micromass GV Sector 54 multicollector mass spectrometer. In Johannesburg, reproducibilities of $1 \%$ and $0.014 \%$ were obtained for the ${ }^{87} \mathrm{Rb} /{ }^{86} \mathrm{Sr}$ and ${ }^{87} \mathrm{Sr} /{ }^{86} \mathrm{Sr}$ ratio measurements, respectively. All ${ }^{87} \mathrm{Sr} /{ }^{86} \mathrm{Sr}$ ratios were normalized to an ${ }^{88} \mathrm{Sr} /{ }^{86} \mathrm{Sr}$ ratio of 8.375 . The accuracy of the ${ }^{87} \mathrm{Sr} /{ }^{86} \mathrm{Sr}$ ratio was monitored by replicate analyses of the NBS SRM987 Sr standard $\left(0.710190 \pm 24 ; 2 \sigma_{\mathrm{m}}\right.$ on 6 measurements $)$. For the $\mathrm{Nd}$ isotopic analysis, the ${ }^{143} \mathrm{Nd} /{ }^{144} \mathrm{Nd}$ ratio was normalized to a ${ }^{146} \mathrm{Nd} /{ }^{144} \mathrm{Nd}$ ratio of 0.7219 . The accuracy of the ${ }^{143} \mathrm{Nd} /{ }^{144} \mathrm{Nd}$ ratio was monitored by replicate analyses of the La Jolla Nd standard, which yielded an average value of 0.511835 . In Brussels, the average ${ }^{87} \mathrm{Sr} /{ }^{86} \mathrm{Sr}$ ratio of the NBS SRM987 standard and ${ }^{143} \mathrm{Nd} /{ }^{144} \mathrm{Nd}$ ratio of the La Jolla $\mathrm{Nd}$ standard during the period of analyses were $0.710255 \pm 10\left(2 \sigma_{\mathrm{m}}\right.$ on 20 measurements) and $0.511854 \pm 8$ (on 7 measurements), respectively.

\section{References}

Alt, J.C., Honnorez, J., 1984. Alteration of the upper oceanic crust, DSDP site 417: mineralogy and chemistry. Contrib. Mineral. Petrol. 87, 149-169.
Andreoli, M.A.G., Hart, R.J., 1987. Explosive KREEP-norite from Namaqualand, South Africa, with implications for the Sudbury Irruptive. In: Proceedings of the International Workshop on Cryptoexplosions and Catastrophes in the Geological Record, Section A, Parys, South Africa, pp. 1-8.

Barnes, S.J., 1986. The effect of trapped liquid crystallization on cumulus mineral composition in layered intrusions. Contrib. Mineral. Petrol. 93, 523-531.

Bédard, J.H., 1994. A procedure for calculating the equilibrium distribution of trace elements among the minerals of cumulate rocks, and the concentrations of trace elements in the coexisting liquids. Chem. Geol. 118, 143-153.

Boer, R.H., Meyer, F.M., Cawthorn, R.G., 1994. Stable isotopic evidence for crustal contamination and desulfidation of the cupriferous Koperberg Suite, Namaqualand, South Africa. Geochim. Cosmochim. Acta 58, 2677-2688.

Bologne, G., Duchesne, J.C., 1991. Analyse des roches silicatées par spectrométrie de fluorescence X: précision et exactitude. Belgian Geol. Survey Prof. Paper 249, 1-11.

Brandriss, M.E., Cawthorn, R.G., 1996. Formation of anorthosite and leucotonalite during magma hybridization in the Koperberg Suite of Namaqualand, South Africa. S. Afr. J. Geol. 99, 135152.

Cawthorn, R.G., 1996. Models for incompatible trace-element abundances in cumulus minerals and their application to plagioclase and pyroxenes in the Bushveld Complex. Contrib. Mineral. Metrol. 123, 109-115.

Cawthorn, R.G., Meyer, F.M., 1993. Petrochemistry of the Okiep Copper District basic intrusive bodies, Northwestern Cape province, South Africa. Econ. Geol. 88, 590-605.

Clifford, T.N., Gronow, J., Rex, D.C., Burger, A.J., 1975. Geochronological and petrogenetic studies of high-grade metamorphic rocks and intrusives in Namaqualand, South Africa. J. Petrol. 16, 154-188.

Clifford, T.N., Stumpfl, E.F., Burger, A.J., McCarthy, T.S., Rex, D.C., 1981. Mineral-chemical and isotopic studies of Namaqualand granulites, South Africa: a Grenville analogue. Contrib. Mineral. Petrol. 77, 225-250.

Clifford, T.N., Barton, E.S., Retief, E.A., Rex, D.C., Fanning, C.M., 1995. A crustal progenitor for the intrusive anorthosite-charnockite kindred of the cupriferous Koperberg Suite, O'okiep district, Namaqualand, South Africa; new isotope data for the country rocks and the intrusives. J. Petrol. 16, 154-188.

Clifford, T.N., Barton, E.S., Stern, R.A., Duchesne, J.C., 2004. U-Pb zircon calendar for Namaquan (Grenville) crustal events in the granulite-facies terrane of the O'okiep Copper District of South Africa. J. Petrol. 45, 669-691.

Condie, K.C., 1993. Chemical composition and evolution of the upper continental crust: contrasting results from surface samples and shales. Chem. Geol. 104, 1-37.

Conradie, J.A., Schoch, A.E., 1986. Petrographical and petrochemical aspects of the Koperberg Suite, South Africa-an analogy to massif-type anorthosites? Precambrian Res. 31, 157-188.

Conradie, J.A., Schoch, A.E., 1988. Rare earth element geochemistry of an anorthosite-diorite suite, Namaqua mobile belt, South Africa. Earth Planet. Sci. Lett. 87, 409-422.

Cornell, D.H., Kröner, A., Humphreys, H., Griffin, G., 1990. Age of origin of the polymetamorphosed Copperton Formation, NamaquaNatal Province, determined by single grain zircon $\mathrm{Pb}-\mathrm{Pb}$ dating. S. Afr. J. Sci. 93, 709-716.

Demaiffe, D., Hertogen, J., 1981. Rare earth element geochemistry and strontium isotopic composition of a massif-type anorthositic- 
charnockitic body: the Hidra massif (Rogaland, SW Norway). Geochim. Cosmochim. Acta 45, 1545-1561.

Duchesne, J.C., 1978. Quantitative modeling of Sr, Ca, Rb and K in the Bjerkrem-Sogndal layered lopolith (SW Norway). Contrib. Mineral. Petrol. 66, 175-184.

Duchesne, J.C., 1990. Origin and evolution of monzonorites related to anorthosites. Schweiz. Mineral. Petrogr. Mitt. 70, 189-198.

Duchesne, J.C., Hertogen, J., 1988. Le magma parental du lopolithe de Bjerkreim-Sokndal (Norvège méridionale). C.R. Acad. Sci. Paris 306, 45-48.

Duchesne, J.C., Wilmart, E., 1997. Igneous charnockites and related rocks from the Bjerkreim-Sokndal layered intrusion (Southwest Norway): a jotunite (hypersthene monzodiorite)-derived A-type granitoid suite. J. Petrol. 38, 337-369.

Duchesne, J.C., Roelandts, I., Demaiffe, D., Hertogen, J., Gijbels, R., De Winter, J., 1974. Rare-earth data on monzonoritic rocks related to anorthosites and their bearing on the nature of the parental magma of the anorthositic series. Earth Planet. Sci. Lett. 24, 325-335.

Duchesne, J.C., Wilmart, E., Demaiffe, D., Hertogen, J., 1989. Monzonorites from Rogaland (Southwest Norway): a series of rocks coeval but not comagmatic with massif-type anorthosites. Precambrian Res. 45, 111-128.

Duchesne, J.C., Berza, T., Liégeois, J.P., Vander Auwera, J., 1998. Shoshonitic liquid line of descent from diorite to granite: the Late Precambrian post-collisional Tismana pluton (South Carpathians, Romania). Lithos 45, 281-304.

Duchesne, J.C., Liégeois, J.P., Vander Auwera, J., Longhi, J., 1999. The crustal tongue melting model and the origin of massive anorthosites. Terra Nova 11, 100-105.

Frost, B.R., Frost, C.D., Hulsebosch, T.P., Swapp, S.M., 2000. Origin of the charnockites of the Louis Lake Batholith, Wind River Range, Wyoming. J. Petrol. 41, 1759-1776.

Geringer, G.J., Schoch, A.E., Sukhanov, M., Zhuravlev, D., 1998. Geochemical and isotopic characteristics of different types of anorthosite in the Namaqua mobile belt, South Africa. Chem. Geol. $145,17-46$.

Ghiorso, M.S., Sack, R.O., 1995. Chemical mass balance in magmatic processes. IV. A revised and internally consistent thermodynamic model for the interpolation and extrapolation of liquid-solid equilibria in magmatic systems at elevated temperatures and pressures. Contrib. Mineral. Petrol. 119, 197-212.

Gibson, R.L., Robb, L.J., Kisters, A.F.M., Cawthorn, R.G., 1996. Regional setting and geological evolution of the Okiep Copper District, Namaqualand, South Africa. S. Afr. J. Geol. 99, 107-120.

Harris, N.B.W., Inger, S., 1992. Trace element modelling of pelitederived granites. Contrib. Miner. Petrol. 110, 46-56.

Hartnady, C.J.H., Joubert, P., Stowe, C.W., 1985. Proterozoic crustal evolution in southwestern Africa. Episodes 8, 236-244.

Henderson, P., 1982. Inorganic Geochemistry. Pergamon Verlag, Oxford, p. 353.

Irvine, T.N., 1980. Magmatic infiltration metasomatism, doublediffusive fractional crystallization, and adcumulus growth in the Muskox intrusion and other layered intrusions. In: Hargraves, R.B. (Ed.), Physics of Magmatic Processes. Princeton University Press, Princeton, pp. 325-384.

Irvine, T.N., 1982. Terminology of layered intrusions. J. Petrol. 23, $127-162$.

Kisters, A.F.M., Potgieter, J.E., Charlesworth, E.G., Anhaeusser, C.R., Gibson, R.L., Watkeys, M.K., 1994. Emplacement features of cuprigenous noritoids in the Okiep Copper District, Namaqualand, South Africa. Explor. Mining Geol. 3, 297-310.
Lachenbruch, A.H., Sass, J.H., Galanis Jr., S.P., 1985. Heat flow in southernmost California and the origin of the Salton trough. J. Geophys. Res. 90, 6709-6736.

Liégeois, J.P., Berza, T., Tatu, M., Duchesne, J.C., 1996. The Neoproterozoic Pan-African basement from the Alpine Lower Danubian nappe system (South Carpathians, Romania). Precambrian Res. 80, 281-301.

Liégeois, J.P., Navez, J., Hertogen, J., Black, R., 1998. Contrasting origin of post-collisional high-K calc-alkaline and shoshonitic versus alkaline and peralkaline granitoids. Lithos 45, 1-28.

Liégeois, J.P., Latouche, L., Boughara, M., Navez, J., Guiraud, M., 2003. The LATEA metacraton (Central Hoggar, Tuareg shield, Algeria): behaviour of an old passive margin during the PanAfrican orogeny. J. Afr. Earth Sci. 37, 161-190.

Lombaard, A.F., the exploration staff of the O'okiep Copper Company Limited, 1986. The copper deposits of the OKiep District, Namaqualand. In: Anhaeusser, C.R., Maske, S. (Eds.), Mineral Deposits of Southern Africa, vol. II. Geological Society of South Africa, pp. 1421-1445.

Lombaard, A.F., Schreuder, F.J.G., 1978. Distribution patterns and general geological features of steep structures, megabreccias and basic rocks in the Okiep Copper District. In: Verwoerd, W.J. (Ed.), Mineralization in Metamorphic Terranes, special volume 4. Geological Society of South Africa, pp. 297-310.

Longhi, J., 2005. A mantle or mafic crustal source for Proterozoic anorthosites? Lithos 83, 183-198.

Longhi, J., Vander Auwera, J., Fram, M., Duchesne, J.C., 1999. Some phase equilibrium constraints on the origin of Proterozoic (massif) anorthosites and related rocks. J. Petrol. 40, 339-362.

Martin, H., 1987. Petrogenesis of Archaean trondhjemites, tonalites and granodiorites from Eastern Finland: major and trace element geochemistry. J. Petrol. 28, 921-953.

McCarthy, T.S., 1976. Chemical interrelationships in a low-pressure granulite terrain in Namaqualand, South Africa, and their bearing on granite genesis and the composition of the lower crust. Geochim. Cosmochim. Acta 40, 1057-1068.

McCarthy, T.S., 1977. Geochemical Studies of Selected Granitic Terrains in South Africa. University of the Witwatersrand, Johannesburg, p. 268.

McIver, J.R., McCarthy, T.S., Packham, B.d.V., 1983. The copperbearing basic rocks of Namaqualand. Miner. Deposita 18, $135-160$.

Miller, C.F., 1985. Are strongly peraluminous magmas derived from pelitic sedimentary sources? J. Geol. 93, 673-689.

Oliveira, E.P., Tarney, J., 1995. Genesis of the Precambrian copper-rich Caraiba hypersthenite-norite complex, Brazil. Miner. Deposita 30, 351-373.

Patiño-Douce, A.E., Johnston, A.D., 1991. Phase equilibria and melt productivity in the pelitic system: implications for the origin of peraluminous granitoids and aluminous granulites. Contrib. Miner. Petrol. 107, 202-218.

Peccerillo, R., Taylor, S.R., 1976. Geochemistry of Eocene calcalkaline volcanic rocks from the Kastamonu area, northern Turkey. Contrib. Miner. Petrol. 58, 489-502.

Raith, J.G., 1995. Petrogenesis of the Concordia Granite Gneiss and its relation to W-Mo mineralization in western Namaqualand, South Africa. Precambrian Res. 70, 303-335.

Reid, D.L., Barton, E.S., 1983. Geochemical characterization of granitoids in the Namaqualand geotraverse. Spec. Publ. Geol. Soc. S. Afr. 10, 67-82.

Robb, L.J., Armstrong, R.A., Waters, D.J., 1999. The history of granulite-facies metamorphism and crustal growth from single zir- 
con U-Pb geochronology: Namaqualand, South Africa. J. Petrol. 40, 1747-1770.

Roberts, M.P., Clemens, J.D., 1993. Origin of high-potassium, calcalkaline, I-type granitoids. Geology 21, 825-828.

Robins, B., Tumyr, O., Tysseland, M., Garmann, L.B., 1997. The Bjerkreim-Sokndal Layered Intrusion, Rogaland, S.W. Norway: evidence from marginal rocks for a jotunite parent magma. Lithos 39, 121-133.

Rollinson, H., 1993. Using Geochemical Data: Evaluation, Presentation, Interpretation. Longman Scientific and Technical, p. 352.

Rona, P.A., Klinkhammer, G., Nelsen, T.A., Trefry, J.H., Elderfield, H., 1986. Black smokers, massive sulphides and vent biota at the Mid-Atlantic Ridge. Nature 321, 33-37.

Schiellerup, H., Lambert, D.D., Pretsvik, T., Robins, B., McBride, J.S., Larsen, R.B., 2000. Re-Os isotopic evidence for a lower crustal origin of massif-type anorthosites. Nature 405, 781-784.

Schoch, A.E., Conradie, J.A., 1990. Petrological and mineralogical relationships in the Koperberg Suite, Namaqualand, South Africa. Am. Mineral. 75, 27-36.

Strauss, C.A., 1941. The geology, copper-ore deposits and ground water hydrology of the area around Springbok and O'okiep, Namaqualand. D.Sc. Thesis. University of Stellenbosh, 166 pp.

Streckeisen, A., 1974. How should charnockitic rocks be named? In: Bellière, J., Duchesne, J.C. (Eds.), Géologie des domaines cristallins. Soc. Géol. de Belgique, Centenaire, pp. 349-360.

Stumpfl, E.F., Clifford, T.N., Burger, A.J., Van Zyl, D., 1976. The Copper deposits of the O'okiep District, South Africa: new data and concepts. Miner. Deposita 11, 46-70.

Teyssier, C., Tikoff, B., 1998. Strike-slip partitioned transpression of the San Andreas fault system: a lithospheric-scale approach. In: Holdworth, R.E., Strachan, R.A., Dewey, J. (Eds.), Continental Transpressional and Transtensional Tectonics, vol. 135. Geological Society of London Special publication, pp. 143-158.

Thomas, R.J., Agenbacht, A.L.D., Cornell, D.H., Moore, J.M., 1994. The Kibaran of southern Africa: Tectonic evolution and metallogeny. Ore Geol. Rev. 9, 131-160.

Van Zwieten, A.J.M., McCarthy, T.S., Cawthorn, R.G., 1996. A petrogenetic model for the Koperberg Suite: evidence from the Jubilee Mine, Namaqualand, South Africa. S. Afr. J. Geol. 99, 121-134.

Van Zyl, D., 1978. A petrological approach towards the ore-bearing potentialities of the Okiep basic intrusives in Namaqualand. In:
Verwoerd, W.J. (Ed.), Mineralization in Metamorphic Terranes, Special Publication, vol. 4. Geological Society of South Africa, pp. 323-329.

Vander Auwera, J., Longhi, J., 1994. Experimental study of a jotunite (hypersthene monzodiorite): constraints on the parent magma composition and crystallization conditions ( $\mathrm{P}, \mathrm{T}, \mathrm{fO} 2)$ of the Bjerkreim-Sokndal layered intrusion. Contrib. Miner. Petrol. 118, 60-78.

Vander Auwera, J., Bologne, G., Roelandts, I., Duchesne, J.C., 1998a. Inductively coupled plasma-mass spectrometry (ICP-MS) analysis of silicate rocks and minerals. Geol. Belgica 1, 4953.

Vander Auwera, J., Longhi, J., Duchesne, J.C., 1998b. A liquid line of descent of the jotunite (hypersthene monzodiorite) suite. J. Petrol. 39, 439-468.

Vander Auwera, J., Longhi, J., Duchesne, J.C., 2000. Pressure effects on the partitioning of $\mathrm{Sr}$ between pagioclase and melt and of $\mathrm{Cr}$ between low-Ca pyroxene and melt: implications for anorthosite petrogenesis. Earth Planet. Sci. Lett. 178, 303-314.

Vander Auwera, J., Bogaerts, M., Liégeois, J.P., Demaiffe, D., Wilmart, E., Bolle, O., Duchesne, J.C., 2003. Derivation of the 1.0-0. $9 \mathrm{Ga}$ ferro-potassic A-type granitoids of southern Norway by extreme differentiation from basic magmas. Precambrian Res. 124, $107-148$.

Whalen, J.B., Currie, K.L., Chappell, B.W., 1987. A-type granites: geochemical characteristics, discrimination and petrogenesis. Contrib. Mineral. Petrol. 95, 407-419.

White, V.M., Tapia, M.D.M., Schilling, J.G., 1979. The petrology and geochemistry of the Azores Islands. Contrib. Mineral. Metrol. 69, 201-213.

Wiebe, R.A., 1984. Comingling of magmas in the Bjerkrem-Sogndal lopolith (Southwest Norway): evidence for the compositions of residual liquids. Lithos 17, 171-188.

Wilmart, E., Demaiffe, D., Duchesne, J.C., 1989. Geochemical constraints on the genesis of the Tellnes ilmenite deposit (S. W. Norway). Econ. Geol. 84, 1047-1056.

Wiszniewska, J., Claesson, S., Stein, H.J., Vander Auwera, J., Duchesne, J.C., 2002. The north-eastern Polish anorthosite massifs: petrological, geochemical and isotopic evidence for a crustal derivation. Terra Nova 14, 451-460. 